\title{
Breaking Entry- and Species Barriers: LentiBOOST® Plus Polybrene Enables High Transduction Efficacy of Dendritic Cells and Monocytes by Adenovirus 5
}

Astrid Strack ( $\sim$ astrid.strack@uk-erlangen.de)

Universitätsklinikum Erlangen, Friedrich- Alexander-Universität Erlangen-Nürnberg

Andrea Deinzer

Universitätsklinikum Erlangen, Friedrich- Alexander-Universität Erlangen-Nürnberg

Christian Thirion

SIRION Biotech GmbH

Silke Schrödel

SIRION Biotech GmbH

Jan Dörrie

Universitätsklinikum Erlangen, Friedrich-Alexander- Universität Erlangen-Nürnberg

Tatjana Sauerer

Universitätsklinikum Erlangen, Friedrich-Alexander- Universität Erlangen-Nürnberg

Alexander Steinkasserer

Universitätsklinikum Erlangen, Friedrich- Alexander-Universität Erlangen-Nürnberg

Ilka Knippertz

Universitätsklinikum Erlangen, Friedrich- Alexander-Universität Erlangen-Nürnberg

\section{Research Article}

Keywords: trigger strong immune responses, adenoviruses (HAdVs), serotype5, LentiBOOST Polybrene, dendritic cells (DCs)

Posted Date: August 9th, 2021

DOI: https://doi.org/10.21203/rs.3.rs-701427/v1

License: (c) (i) This work is licensed under a Creative Commons Attribution 4.0 International License.

Read Full License 


\section{Abstract}

Due to their ability to trigger strong immune responses, adenoviruses (HAdVs) in general and the serotype5 (HAdV5) in particular are amongst the most popular viral vectors in research and clinical application. However, efficient transduction using HAdV5 is predominantly achieved in coxsackie and adenovirus receptor (CAR)-positive human cells. In the present study, we used the transduction enhancer LentiBOOST ${ }^{\circledR}$ comprising the polycationic Polybrene to overcome these limitations.

Using LentiBOOST ${ }^{\circledR} /$ Polybrene, we yielded transduction rates higher than $50 \%$ in murine bone marrow derived dendritic cells (BMDCs), while maintaining their cytokine expression profile and their capability to induce T-cell proliferation. In human dendritic cells (DCs), we increased the transduction rate from $22 \%$ in immature (i)DCs or $43 \%$ in mature $(\mathrm{m}) \mathrm{DCs}$ to more than $80 \%$, without inducing cytotoxicity. While expression of specific maturation markers was slightly upregulated using LentiBOOST®/ Polybrene on iDCs, no effect on mDC phenotype or function was observed. Moreover, we achieved efficient HAdV5 transduction also in human monocytes and were able to subsequently differentiate them into proper iDCs and functional mDCs.

In summary, we introduce LentiBOOST $®$ comprising Polybrene as a highly potent adenoviral transduction agent for new in vitro applications in a set of different immune cells in both mice and humans.

\section{Introduction}

A variety of gene delivery techniques have been established over the last decades which can be subdivided into non-viral and viral strategies. The non-viral subclass comprises methods like injection of naked DNA, electroporation, gene gun, sonoporation, magnetofection, and lipoplexes while for the viral subclass lentivirus (LV), herpes simplex virus (HSV), baculovirus, adeno-associated virus (AAV), and human adenovirus (HAdV) have been used for gene transfer ${ }^{1}$. To date, recombinant HAdVs are the most widely used viral vectors for gene therapy, accounting for $18.6 \%$ of vectors used in gene therapy clinical trials, especially in the context of different cancer types, HIV, tuberculosis, Ebola virus, malaria, influenza and lately SARS-CoV-2 ${ }^{1-4}$. In contrast to most other viral vaccines, HAdVs trigger both a robust cytotoxic T-cell response as well as a humoral response which is favourable with regard to the destruction of virusinfected cells, intracellular pathogens, and cancerous cells ${ }^{5-7}$.

The best studied member of the HAdV species is serotype 5 (HAdV5, species HAdV-C) ${ }^{8}$. HAdV5 is characterized by an icosahedral capsid ( $90 \mathrm{~nm}$ in diameter) and a $\sim 35 \mathrm{~kb}$ long double-stranded linear single DNA genome ${ }^{9,10}$. The viral capsid is mainly comprised of three proteins called hexon, penton base, and fibre which interact directly and are held together by cement proteins ${ }^{11,12}$. The primary entry receptor for HAdV 5 is the coxsackievirus and adenovirus receptor (CAR) but also other receptors are utilized by HAdVs, like vascular cell adhesion molecule (VCAM)-1; heparan sulfate proteoplycan (HSPG), major histocompatibility complex (MHC1)-A2, or scavenger receptor A (SR-A) ${ }^{13}$. Hence, HAdV5s can infect a broad range of dividing and non-dividing cells, including immune cells. Therefore HAdV5 is not only a 
very interesting tool to target immune cells in vitro and in vivo for vaccine approaches, but also for gene transfer into cells which are difficult to transfect or electroporate in vitro.

Human monocyte-derived dendritic cells (DCs), which cannot be efficiently transfected using standard transfection reagents or rapidly die after electroporation with DNA, can be transduced with HAd5V vectors in vitro to e.g. analyse cell-type specific functions or for genome editing using CRISPR/ Cas9 ${ }^{14,15}$. Other approaches aim to modify the function of human DCs for the development of new vaccines by overexpressing therapeutic transgenes. However, high titres of HAd5V vectors are often needed to obtain optimal transduction rates in those cells, which are typically associated with higher mortality. Moreover, transduction efficacy using HAdV5 vectors for other primary cell types, like human monocytes or immature murine bone-marrow derived dendritic cells (BMDCs), is often not more than ten percent, even at high titres. The reason for the latter is that HAdV5 is species-specific and naturally does not infect cells of murine origin ${ }^{16}$.

In the light of these barriers, we came up with a transduction enhancer developed by Sirion Biotech, the LentiBOOST ${ }^{\circledR}$ reagent. LentiBOOST ${ }^{\circledR}$ is a highly effective, non-cytotoxic transduction enhancer, originally developed for preclinical and clinical application of lentiviral vectors. It contains two separate components acting receptor-independent and synergistically, the Poloxamer 338, developed by Sirion Biotech for clinical use, and Polybrene. The Poloxamer 388 has been shown to mediate high transduction rates for clinically relevant cell types, including human $\mathrm{CD} 34^{+}$hematopoietic stem cells (HSCs), primary T cells and NK cells, with LVs. The poloxamer is combined with Polybrene, a polycationic substance, which is used to enhance LV transduction efficacy. Currently, LentiBOOST ${ }^{\circledR}$ is used in several clinical trials in the US and Europe up to phase 3, and has been proven to be safe and clinically effective. In 2019, the National Institute of Allergy and Infectious Diseases (NIAID), part of the National Institutes of Health, applied this technology for its lentivirus-based early clinical development of its SCID-X1 trial and was able to develop an enhanced protocol for engineering human $\mathrm{CD} 34^{+}$stem cells.

In the present study, we evaluated the ability of LentiBOOST ${ }^{\circledR}$ mixed with Polybrene to enhance and optimize the transduction efficacy of murine immature (i)BMDCs and mature (m)BMDCs, as well as of human monocytes and immature (i)DCs and mature (m)DCs, with HAd5V. For this purpose, we first assessed the optimal transduction conditions, with respect to the used multiplicity of infection (MOI) of the HAd5V vector, encoding for the green fluorescent protein (GFP), in combination with LentiBOOST ${ }^{\circledR} /$ Polybrene, for iBMDCs, mBMDCs, iDCs and mDCs. Using this optimized transduction protocol, we analysed infected murine and human DCs further, regarding their survival, expression of typical cell surface markers, secretion of cytokines and their ability to prime naïve allogeneic T cells. Subsequently,

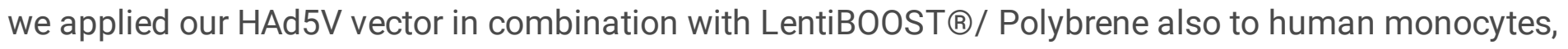
where we observed high transduction rates. Notably, subsequently we were still able to differentiate these transduced monocytes into iDCs and mDCs, displaying a typical phenotype and function, since these generated mDCs were not only able to prime naïve T cells in a mixed lymphocyte reaction (MLR), but 
could also be electroporated with a mRNA coding for a tumour antigen, or loaded with peptide to induce a tumour antigen-specific T-cell response in vitro.

\section{Results}

\section{LentiBOOST® in combination with Polybrene enables transduction of murine BMDCs with HAdV5.}

To overcome the low transduction efficacy due to the lack of CAR expression on target cells, or the species-specificity of the HAdV serotype, we examined the transduction agent LentiBOOST ${ }^{\circledR}$ combined with Polybrene in the context of HAdV transduction, of human and murine primary cells. Therefore, we first transduced murine immature and mature BMDCs with a HAdV5 coding for GFP at MOls from 40 to 500 , either in the presence or absence of LentiBOOST ${ }^{\circledR}$ /Polybrene (LeB/ PB; Fig. 1). As a negative control, cells were either mock-transduced or transduced with a HAdV5, encoding for the renilla firefly luciferase (HAdV5Luc1). As assessed by flow cytometry 48 hours afterwards, percentage of GFP ${ }^{+}$ iBMDCs increased from 2-9\% (MOI40), 3-27\% (MOI100), 5-42\% (MOI200) and 11-54\% (MOI500), using LeB/ PB (Fig. 1a). No significant cytotoxicity was observed, except for the highest MOI used in combination with LeB/ PB (Fig. 1b). Similar results were obtained using mBMDCs (Fig. 1c, d). Here, the percentage of $\mathrm{GFP}^{+}$cells was enhanced by the combination LeB/ PB with increasing MOls, from $3-15 \%$ (MOI40), and from 24-54\% (MOI500), while survival of mBMDCs was unaltered (Fig. 1d). Moreover, LeB/ PB did not affect the morphology of either iBMDCs or mBMDCs (Fig. 1e, f).

In summary, LentiBOOST ${ }^{\circledR}$ combined with Polybrene breaks species-specific HAdV5 infection barriers of murine immature and mature BMDCs, without toxic side effects. As MOls 200 and 500 of HAdV5GFP were shown to be most efficient to transduce immature and mature BMDCs, these were used for the following experiments.

\section{LentiBOOST®/ Polybrene does not alter the phenotype and function of murine BMDCs.}

As DCs rapidly react on environmental changes, we next investigated the influence of the LentiBOOST®/ Polybrene regarding the phenotype and function of immature and mature BMDCs, 48 hours after transduction with HAdV5Luc1 (MOI500) and HAdV5GFP (MOI200 and MOI500), in comparison to cells inoculated with adenovirus alone. Flow cytometric analyses revealed no differences regarding the expression of classical DC cell surface markers, such as CD25, CD80, CD83, CD86 and MHC class II, on iBMDCs (Fig. 2a), compared to cells transduced in the absence of LentiBOOST ${ }^{\circledR} /$ Polybrene (-LeB/ PB). Interestingly, the usage of a GFP encoding adenovirus induced a strong maturation of iBMDCs, independent of the used $\mathrm{MOI}$, which was not observed for firefly luciferase encoding HAdV5 (HAdV5Luc1) transduced cells, or mock-treated cells (Fig. 2a). Expression of CD25, CD80 and CD86, on the cell surface of mature BMDCs, was not affected by LentiBOOST ${ }^{8} /$ Polybrene (Fig. 2b). Induction of BMDCs 
maturation was accompanied by the secretion of pro-inflammatory cytokines, like MCP-1, TNF-a, IL-6 and IFN- $\beta$, while mock- or HAdV5Luc1 treated iBMDCS produced only very low -, or almost no cytokines (see Supplementary Fig. S1a online). Additionally, HAdV5GFP + LeB/ PB led to an increased production of IL-6 and IFN- $\beta$ by iBMDCs, when compared with HAdV5GFP - LeB/ PB (see Supplementary Fig. S1a online). A slightly altered secretion of MCP-1, IL-1 $a$ and IL- 6 was also observed for mBMDCs (see Supplementary Fig. S1b online).

To address the functionality of these differentially treated mBMDCs, mixed lymphocyte reactions (MLR) were performed (Fig. 3a, b). Here, LentiBOOST $® /$ Polybrene ("Mock" + LeB/ PB) or in combination with HAdV5Luc1 (MOI 200 and MOI 500), did not affect the capability of mBMDCs to prime allogeneic $T$ cells. By contrast, transduction of mBMDCs with HAdV5GFP in the presence of LentiBOOST ${ }^{8} /$ Polybrene led to a stronger proliferation of T cells at a DC:T cell ratio of 1:10 (1.3 fold MOI200/ 1.7 fold MOI500), and 1:33 (1.5 fold MOI200/ 1.7 fold MOI500). This effect was not observed for HAdV5GFP transduced BMDCs missing the LeB/ PB. Next, we examined the portfolio of classical T cell derived cytokines in the supernatants of the co-cultures. We observed no differences between BMDCs transduced in the absence (-LeB/ PB) or presence (+ LeB/ PB), to induce classical Th1/ Th2 associated cytokines IL-2 and IL-6 (Fig. 3c), Th1 cytokines IFN-y and TNF-a (Fig. 3d), Th2 cytokines IL-4 and IL-13 (Fig. 3e), or Th17 cytokines IL17A and IL-22 (Fig. 3f).

Taken together, phenotype and function of BMDCs are not altered by LentiBOOST®/ Polybrene, although a slight increase (i) in the secretion of pro-inflammatory cytokines by iBMDCs, and (ii) the capability of mBMDCs to prime T cells was observed, partially depending on the transgene encoded by the adenovirus.

\section{LentiBOOST®/ Polybrene increases transduction efficiency of human monocyte-derived DCs at low HAdV5 virus titres.}

Although lacking CAR expression, human DCs can be transduced with HAdV5 at high titres > MOI500. Since high virus titres are accompanied by toxicity, followed by loss of function, we next assessed if LentiBOOST ${ }^{\circledR} /$ Polybrene can improve HAdV5-mediated gene delivery to human DCs. Flow cytometric analyses, 48 hours after transduction of immature (i)DCs and mature (m)DCs with HAdV5GFP at different MOls, revealed that a $\mathrm{MOI}$ of 10 is already sufficient to infect $\sim 60 \%$ of iDCs, while without LentiBOOST ${ }^{\circledR} /$ Polybrene only $1 \%$ of cells were GFP-positive (Fig. 4a). Using a MOI of 100 or $200,>80 \%$ of cells were GFP-positive, while only $22 \%$ or $42 \%$ of cells were transduced in the absence of LentiBOOST ${ }^{\circledR} /$ Polybrene, respectively. Toxicity was moderate, with a decrease of living cells from 9680\% (MOI100), or 92-65\% (MOl200; Fig. 4b). Transduction efficacy of mDCs increased from 1-20\% (MOI 10), 1-49\% (MOI 20), 13-67\% (MOI40), 43-84\% (MOI100) and from 63-84\% (MOI200), using the LeB/ PB (Fig. 4c). Again, survival of mDCs was not impaired (Fig. 4d). Moreover, iDCs and mDCs showed a similar morphology and cell clustering may be increased in the presence of LentiBOOST $® /$ Polybrene, independent of the adenovirus (Fig. $4 \mathrm{e}$ and f). Overall, LentiBOOST ${ }^{\circledR}$ combined with Polybrene efficiently transduces iDCs and mDCs, using a MOI of 10 or 40, respectively, with an optimal MOI of 100 . 


\section{LentiBOOST®/ Polybrene induces maturation of immature human DCs but does not alter DC function.}

Next we analysed, if LentiBOOST $® /$ Polybrene and the observed enhanced infection rate influence the phenotype and function of human DCs. Thus, iDCs and mDCs, either mock-treated, or transduced with HAdV5Luc1 (MOI200), or HAdV5GFP (MOI 100 and 200), were analysed by flow cytometry, 48 hours post infection. In contrast to murine iBMDCs, human iDCs exposed to LentiBOOST®/ Polybrene showed a significant upregulation of maturation markers CD83 and CD86, while CD25, CD80 and HLA-DR were not statistically altered (Fig. 5a). For HAdV5GFP the median was even higher when compared to mock- or HAdV5Luc1 controls. Interestingly, we observed almost no differences regarding the cytokine production, except for IL-8, which was clearly upregulated in the presence of the LentiBOOST $\AA /$ Polybrene (see Supplementary Fig. S2a online). However, inoculation of mDCs with LentiBOOST®/ Polybrene did not alter their expression profiles in comparison to control mDCs (Fig. 5b), nor changed their cytokine production (see Supplementary Fig. S2b online). Moreover, median fluorescence intensity was strongly increased for all analysed cell surface markers, including CD80, CD83, CD86 and HLA-DR, when compared with LentiBOOST ${ }^{\circledR} /$ Polybrene treated iDCs.

Next, to analyse the mDCs-mediated allogeneic T cell proliferation, MLR assays were performed. Results revealed that neither LentiBOOST $\AA /$ Polybrene nor the amount of adenovirus used influenced the $T$ cell stimulatory capacity of mDCs (Fig. 6a, b). When analysing the supernatants derived from the DC - T cell co-cultures for their content of classical T-cell derived cytokines, like IL-2, IL-6, IFN-y, IL-5, IL-13 and IL-22, no differences between $+\mathrm{LeB} / \mathrm{PB}$ and $-\mathrm{LeB} / \mathrm{PB}$ were observed (Fig. 6c-f). However, co-cultures of T cells with HAdV5GFP transduced mDCs showed reduced levels of IL-5, IL-6, IL-13 and IL-22, independent of the LentiBOOST ${ }^{\circledR} /$ Polybrene.

Taken together, HAdV5-mediated gene transfer, in the presence of LentiBOOST®/ Polybrene, does not significantly interfere with the DC phenotype and their function.

\section{Efficient HAdV5-mediated gene transfer into monocytes by LentiBOOST®/ Polybrene allows for proper subsequent iDCs and mDCs differentiation.}

Similar to murine BMDCs, human monocytes are poorly permissive to HAdV5, even at high titres. Hence, we transduced freshly isolated human monocytes (see Supplementary Fig. S3a online) with HAdV5GFP at a $\mathrm{MOI}$ of 100 or 200 , either in the absence or presence of LentiBOOST $\AA /$ Polybrene. Twenty-four hours afterwards, cells were analysed by flow cytometry. As depicted in Fig. 7a, the transduction efficacy increased from 7-36\% (MOI100) and 15-36\% (MOI200), when applying LentiBOOST $8 /$ Polybrene to the cell culture. Notably, mean GFP expression was upregulated from 227 to 3591 (MOl100) and 588 to 4264 (MOI200), while no toxicity (Fig. 7a), no changes in the composition of monocyte subpopulations (see 
Supplementary Fig. S3b online), or cytokine secretion (see Supplementary Fig. S4a online), were observed. Next, transduced monocytes were differentiated into iDCs and mDCs and analysed regarding their phenotype and function. Flow cytometric analyses of day four iDCs, differentiated from transduced monocytes, using IL-4 and GM-CSF, revealed no loss of GFP positivity, in comparison to monocytes, although median values were diminished (Fig. 7b). Again, survival of cells was shown to be $>92 \%$. Further exploration of these cells displayed a typical immature DC-phenotype regarding the expression of CD25, CD80, CD83, CD86 and HLA-DR, in comparison to the "Mock"-control, independent of the presence or absence of LentiBOOST ${ }^{\circledR} /$ Polybrene (Fig. 7c). In addition, cytokine profiles were unaltered (see Supplementary Fig. S4b online).

Next, mDCs were generated from these iDCs by adding a maturation cocktail, consisting of IL-1 $1 \beta$, IL-6, $\mathrm{PGE}_{2}$ and TNF- $\alpha$ for another 24 hours. While GFP expression and survival of those cells was comparable to iDCs shown before (Fig. 7d), matured DCs highly upregulated typical DC maturation markers, such as CD25, CD80, CD83, CD86 and HLA-DR (Fig. 7e). Importantly, the LentiBO0ST $® /$ Polybrene did not affect this maturation process. However, exposure of monocytes to HAdV5GFP alone led to a 2.6- (MOI100) or 2.4 (MOI200) fold increased expression of CD25 on mDCs, which was not observed when LeB/ PB was present during transduction. Moreover, transduction with HAdV5GFP affected upregulation of CD83 in a virus concentration dependent manner, which in contrast to CD25 was independent of the usage of LentiBOOST ${ }^{\circledR} /$ Polybrene. Interestingly, in the supernatants of mock-treated cells, without LeB/ PB, we found strongly increased IL-6, TNF- $a$ and MCP-1 concentrations (see Supplementary Fig. S4c online).

Finally, we investigated the function of these mDCs, using an allogeneic MLR assay and in addition we used them to prime autologous T cells, in a tumour-antigen-specific manner (Fig. 8). Regarding MLR assays, mock- and HAdV5GFP-treated monocytes $-/+\mathrm{LeB} / \mathrm{PB}$, differentiated into mDCs were co-cultured with allogeneic $T$ cells at different ratios, before $T$ cell proliferation was assessed by thymidine incorporation (Fig. 8a). Although we observed a HAdV5GFP- and virus concentration- mediated effect regarding the capacity of mDCs to prime naïve T cells, no LentiBOOST ${ }^{\circledR} /$ Polybrene dependent alterations were observed. In addition, transduced cells ( $/ /+\mathrm{LeB} / \mathrm{PB})$, were still able to induce a high and efficient T-cell proliferation accompanied by an unaltered IL-2 secretion (Fig. 8a, b).

To prime autologous T cells, mDCs were additionally electroporated with RNA coding for the tumour antigen MelanA (MelA) or loaded with a MelA specific peptide. Survival of DCs four hours after electroporation varied from 40-60\% (Fig. 8C). Next, MelA peptide loaded, MelA RNA or control electroporated ("no RNA") DCs, were used to stimulate bulk autologous CD8 ${ }^{+} \mathrm{T}$ cells for one week, before the fraction of MelA-specific T cells was determined by tetramer-staining. As shown in Fig. 8d and S5 (see Supplementary Information online), MelA electroporated and MelA-peptide loaded DCs induced antigenspecific CD8 ${ }^{+} T$ cells, compared to control DCs ("no RNA"), even when they had been transduced with HAdV5 and treated with LentiBOOST ${ }^{\circledR} /$ Polybrene before. Noteworthy, the numbers of MelA $\mathrm{CD}^{+} \mathrm{T}^{+}$cells vary in a donor-dependent manner (see Supplementary Fig. $\mathrm{S} 5$ online), which is rather common when cells derived from different healthy donors are used. However, these data clearly indicate that 
LentiBOOST ${ }^{\circledR} /$ Polybrene does not impair the capability of DCs to express, process, and present a tumour specific antigen in an MHC-class I-restricted manner.

In summary, using LentiBOOST $\AA$ / Polybrene not only allows for the highly efficient transduction of human monocytes with HAdV5, but additionally for the differentiation into iDCs and functional mDCs.

\section{Discussion}

Using directed viral gene transfer to treat human diseases, gene therapy holds the potential to revolutionize medicine ${ }^{1}$. Currently, HAdV vectors attract tremendous attention in the context of newly developed vaccines against SARS-COV2. Amongst those that have been already applied and proven safe and highly effective, some are based on a chimpanzee adenovirus (AZD1222, Jenner Institute/ AstraZeneca/ University of Oxford), or on human adenovirus types 5 and - 26 (Ad5-nCoV, Cansino Biologics/Beijing Institute of Biotechnology; JNJ-78436735 [Ad26]; Janssen Pharmaceutical Companies of Johnson \& Johnson) ${ }^{17}$. However, usage of HAdV5 vectors is limited regarding the transduction of cells lacking appropriate receptors on their cell surface (e.g. monocytes) or species-specificity (e.g. murine cells like BMDCs) ${ }^{16,18}$. In the past, several techniques have been developed to promote virus-mediated gene delivery comprising (i) physical methods, (ii) genetic bioengineering of viruses, and (iii) chemical methods, namely material additives ${ }^{19}$. Amongst these, polymers are the most extensively studied delivery systems for viral vectors like HAdVs ${ }^{19}$ to increase the efficiency of HAdV-mediated gene transfer into epithelial and endothelial- as well as mesenchymal stem cells ${ }^{20,21}$. Besides others, the cationic polymer polybrene is a common enhancer of viral delivery in vitro due to its cost efficiency and its simple and safe handling ${ }^{19,22}$. Poloxamers, on the other hand, are commercially available, FDA-approved, thermoresponsive triblock copolymers, consisting of two blocks of hydrophilic poly(ethylene oxide) (PEO) and one block of hydrophobic poly(propylene oxide) (PPO) ${ }^{19}$. Poloxamer 338 (PE0141-PPO44-PE0141) has been described to efficiently enhance LV delivery in T cells ${ }^{19}$. In combination with polybrene, Höfig and colleagues reported a further elevated transduction efficacy, which was explained by the distinct modes of each adjuvant, polybrene compensating electrostatic repulsion and poloxamer 338 fluidization the membrane ${ }^{19,23}$. LentiBOOST ${ }^{\circledR} /$ Polybrene by Sirion Biotech is such a two-component transduction enhancer and has already been used to efficiently promote LV transduction in vitro to generate melanoma-specific human T cells for cancer immunotherapy ${ }^{24}$. In the present study we used LentiBOOST $\AA /$ Polybrene to transduce not only murine BMDCs, but also human monocyte-derived DCs as well as monocytes, efficiently with HAdV5 in vitro to overcome the barriers of receptor-dependency and species-specificity. In accordance with previous published data, we report here that even at high virus titres (MOI 200 and MOI 500) transduction efficacy for iBMDCs reached only approx. 5-11\%, and $13 \%$ and $24 \%$, for mBMDCs, respectively. While alternative HAdV receptors VCAM- 1 and SR-A are constitutively expressed by bone marrow-derived cells ${ }^{25,26}, \mathrm{MHC}$ class molecules are upregulated upon maturation of BMDCs, which might contribute to the higher infection rate. Nevertheless, a much higher transduction efficacy is the prerequisite of success for most in vitro and in vivo approaches. Using LentiBOOST $\circledast /$ Polybrene transduction efficacy increased to $54 \%$ for immature and mature BMDCs, without any serious 
toxicity, change in phenotype and function of these cells. Interestingly, in comparison to HAdV5Luc1 the HAdV vector containing the green fluorescent protein (GFP) induced maturation of iBMDCs independent of the LentiBOOST $\AA /$ Polybrene, while for mBMDCs no differences in the maturation status were observed. Moreover, GFP-transduced cells showed a higher capacity to induce T cell proliferation. GFP was detected in 1961 as a by-product of the extraction of aequorin from the Auquorea victoria jellyfish 27 , and since then it has been developed further and is still widely used to label cells or to track gene expression. Of notice, several papers described an immunogenic effect of GFP, being slightly immunogenic in C57BL/6 mice and exhibiting a higher immunogenicity in BALB/c mice ${ }^{28-30}$. However, a later study postulated that when used as an intracellular gene reporter, GFP is processed in the host cell followed by presentation at the cell's surface via MHC class I molecules, leading to an antigen-specific recognition by cytotoxic T-lymphocyte ${ }^{27}$. Similarly, in human DCs, GFP expression was shown to act as an "adjuvant" to enhance T-cell immunity to the melanoma tumour antigen MART1 and to expand multiple $\mathrm{CD} 4^{+}$and $\mathrm{CD} 8^{+} \mathrm{T}$ cell clones ${ }^{31}$.

Within in this study, human iDCs transduced with HAdV5GFP showed only an enhanced expression of maturation markers CD80, CD83, CD86 and HLA-DR in comparison to control cells, whereas no differences were observed for mDCs. Moreover, neither the transfer of GFP into mDCs, nor the use of LentiBOOST ${ }^{\circledR} /$ Polybrene resulted in an altered capability of mDCs to stimulate T cells. Merely T cells cocultivated with HAdV5GFP-transduced mDCs produced less Th2-cytokines IL-5, IL-6 and IL-13, thereby in part supporting the postulated immunogenicity of GFP for human DCs reported by Francesca Re and colleagues ${ }^{31}$.

Although Polybrene has been used to improve LV-mediated gene delivery to murine and human DCs, to the best of our knowledge no publication addressed the maturation status of DCs treated with Polybrene only ${ }^{32,33}$. This applies also to the LentiBOOST ${ }^{\circledR}$, the Poloxamer 338 , which in this study has been used for the first time in combination with Polybrene to improve HAdV-mediated gene transfer into DCs and monocytes. Notably, LentiBOOST ${ }^{\circledR} /$ Polybrene increased HAdV-mediated GFP gene transfer into iDCs approximately 4-fold (MOI 100) and 2-fold (MOI 200), to more than $80 \%$ GFP positive cells. Interestingly, this high infection rate is not accompanied by a maturation signal as confirmed by the mock- and HAdV5Luc1 controls.

In a last set of experiments we efficiently transduced human monocytes with HAdV5 followed by proper differentiation of these cells into immature and mature DCs. Usually, Adenoviruses infect human CD14 ${ }^{+}$ monocytes ineffectively, mainly due to the lack of appropriate expression of integrins av $\beta 3$ and av $\beta 5^{34}$, which are the receptors for HAdV5 on these cells ${ }^{35,36}$. Using LentiBOOST ${ }^{\circledR} /$ Polybrene in combination with HAdV5GFP increased the number of $\mathrm{GFP}^{+}$monocytes 5.3 fold (MOI 100) and 2.4 fold (MOI 200) to 36.4 percent. Importantly, this GFP expression was stable during differentiation of monocytes to iDCs and $\mathrm{mDCs}$, thereby allowing for a sustained expression of the delivered gene of interest in transduced DCs. 
Finally, we analysed these transduced monocytes differentiated to mDCs with respect to their functionality. Compared to non-transduced cells, these mDCs retained their full capacity to prime allogeneic T cells, independently of the transduction enhancer substance. Notably, when these mDCs were subjected to electroporation with a tumour mRNA encoding MelanA, they endured this stress test and could be used to prime antigen-specific $C D 8^{+} T$ cells. Although $\mathrm{mDC}$ not transduced with HAdV5GFP (+/- LeB/ PB) as monocytes usually induced higher numbers of $\mathrm{CD}^{+} \mathrm{CD}^{+}$MelanA $^{+} \mathrm{T}$ cells, efficiently infected (HAdV5GFP + LeB/ PB) and electroporated cells were still functional and elicited sufficient antigen-specific $T$ cell responses in vitro. However, the capability of T-cell priming varied between donors. This might be due to the different frequencies of MelanA-specific naïve precursors in the blood of PBMCs of healthy donors. Moreover, these differences in T-cell reactivity reveal the heterogeneity of the immunological status of the different donors in general.

Taken together, here we present the transduction enhancer LentiBOOST ${ }^{\circledR} /$ Polybrene as a promising and valuable tool to increase HAdV transduction efficacy, not only of murine and human DCs but also of human monocytes, without compromising the function of those cells. Therefore, using LentiBOOST®/ Polybrene offers new means for promoting HAdV- or LV mediated viral gene transfer in vitro as well as in vivo for future efficient and safe clinical applications.

\section{Methods}

\section{Mice.}

C57BL/ 6 and BALB/c mice were purchased from Charles River/Wiga (Sulzfeld, Germany) and maintained under pathogen-free conditions according to the European Communities Council Directive (86/609/EEC).

\section{Generation of murine bone marrow derived DCs.}

Bone-marrow derived DCs from C57BL/ 6 mice were generated as described previously ${ }^{37}$ and finally resuspended at a density of $2 \times 10^{6}$ cells in $10 \mathrm{ml} \mathrm{R} 10$ medium consisting of RPMl1640, 1\% Penicillin/Streptomycin/L-Glutamine, 2-ME and 10\% heat-inactivated FBS (GE Healthcare), additionally supplemented with GM-CSF supernatant $(1: 10)$ from a cell line stably transfected with the murine GM$\mathrm{CSF}^{38}$. At days 3 and $6,10 \mathrm{ml}$ of fresh R10 supplemented with GM-CSF supernatant (1:10) was added, with removing $50 \%$ of the old cell culture supernatant at day 6 before. Maturation of BMDCs was induced at day 7 by the addition of $0.1 \mathrm{ng} / \mathrm{ml}$ LPS for 20 hours. At day 8 , cells were used for further experiments.

\section{Generation of human monocytes, monocyte-derived DCs and T cells.}


Human peripheral blood mononucleated cells (PBMCs) of healthy donors were isolated from leucocyte reduction system chambers (LRSCs) using density centrifugation as described previously ${ }^{39}$. For the generation of monocytes, cells were seeded in DC-medium consisting of RPMI1640 + 1\% Penicillin/Streptomycin/Glutamate $+1 \%$ heat-inactivated AB serum $+1 \%$ Hepes after removing the nonadherent fraction (NAF). NAF was cryopreserved and stored at $-80^{\circ} \mathrm{C}$ for isolation of $\mathrm{T}$ cells for allogeneic MLR or for antigen-specific T cell priming.

For the generation of DCs from PBMCs, the adherent cell fraction was cultured for 4 days in DC-medium supplemented with $800 \mathrm{IU} / \mathrm{ml}$ (day 0) or $400 \mathrm{IU} / \mathrm{ml}$ (day 3) recombinant human granulocyte macrophage colony-stimulating factor (GM-CSF) and $250 \mathrm{IU} / \mathrm{ml}$ (day 0 and 3) recombinant IL-4 (both Miltenyi Biotec). On day 4, maturation of DCs was induced when indicated by adding a maturation cocktail consisting of $1000 \mathrm{IU} / \mathrm{ml} \mathrm{IL-6}$ (Miltenyi Biotec), $200 \mathrm{IU} / \mathrm{ml} \mathrm{IL-1 \beta}$ (Cell Genix), $10 \mathrm{ng} / \mathrm{ml}$ tumor necrosis factor a (TNF-a; Peptrotech) and $1 \mu \mathrm{g} / \mathrm{ml}$ prostaglandin $\mathrm{E}_{2}\left(\mathrm{PGE}_{2}\right.$; Santa Cruz) for 24 hours before cells were transduced with adenovirus.

\section{Recombinant adenoviruses.}

HAdV5Luc1 and HAdV5GFP are first generation, E1- and E3-deleted, replication-deficient adenoviral vectors. HAdV5Luc1 contains a CMV-firefly luciferase cassette and was kindly provided by D. T. Curiel, Washington University School of Medicine, MO, US. HAdV5GFP was cloned as follows: a gene cassette containing a CMV-GFP sequence was inserted into pShuttle. Virus genomes were obtained by homologous recombination of the corresponding shuttle plasmids indicated above with pAdEasy-1 in E.

coli BJ5183 as described before ${ }^{40}$. Adenovirus particles were produced by transfection of the different Pacl-digested pAd vectors into 293 cells using Lipofectamine 2000. All viruses were amplified in 293 cells and purified by two rounds of $\mathrm{CsCl}$ equilibrium density gradient ultracentrifugation. Verification of viral genomes and exclusion of wild-type contamination were performed by PCR. Physical particle concentration [viral particles $(\mathrm{vp}) / \mathrm{mL}$ ] was determined by OD260 reading and infectious particle concentration was determined by $\mathrm{TCID}_{50}$ assay on 293 cells.

\section{Adenoviral transduction.}

Human monocytes were transduced in either $450 \mu \mathrm{l}$ (6-well plate)/ $3 \mathrm{ml}$ (cell culture dish) DC-medium (for monocytes) or DC-medium supplemented with either $1600 \mathrm{U} / \mathrm{mL} \mathrm{GM-CSF}$ and $500 \mathrm{U} / \mathrm{mL} \mathrm{IL}-4$ (for iDCs). Virus suspension was prepared using $0.5 \mathrm{mg} / \mathrm{ml}$ LentiBOOST ${ }^{\circledR}+4 \mu \mathrm{g} / \mathrm{ml}$ Polybrene (Sirion Biotech) or buffer only, according to the manufacturer's instructions. Adenovirus was added to the cells at a $\mathrm{MOI}$ of 100 or 200 in a volume of $450 \mu$ (6-well plate) or $3 \mathrm{ml}$ (cell culture dish) resulting in a final infection volume of $900 \mu \mathrm{l}$ or $6 \mathrm{ml}$, respectively. After 1.5 hours of incubation at room temperature on a rocker and 2.5 hours at $37^{\circ} \mathrm{C} / 5 \% \mathrm{CO}_{2}, 3 \mathrm{ml}$ (6-well)/ $18 \mathrm{ml}$ (cell culture dish) of DC-medium only (for monocytes) or replenished with cytokines (for iDCs) as described before was added per well. Transduced monocytes 
were either analysed by flow cytometry 24 hours post infection or were differentiated to day 4 iDCs or day $5 \mathrm{mDCs}$, as described before, and then used for further experiments.

For adenoviral transduction of human DCs, $5 \times 10^{5} \mathrm{iDCs}$ or mDCs were seeded into a 24-well plate in a volume of $125 \mu \mathrm{l} \mathrm{DC}$-medium supplemented with either $1600 \mathrm{U} / \mathrm{mL} \mathrm{GM-CSF}$ and $500 \mathrm{U} / \mathrm{mL} \mathrm{IL}-4$ (for iDCs) or the maturation cocktail consisting of $400 \mathrm{U} / \mathrm{ml} \mathrm{IL-}-1 \beta, 2000 \mathrm{U} / \mathrm{ml} \mathrm{IL-}-6,20 \mathrm{ng} / \mathrm{ml} \mathrm{TNF}-\alpha$, and $2 \mu \mathrm{g} / \mathrm{ml}$ $\mathrm{PGE}_{2}$, in addition to IL-4 and GMCSF (for mDCs). Virus suspension was prepared as described before. Virus dilutions were performed in DC-medium without additives at different MOls and added to the cells to a final volume of $250 \mu \mathrm{l}$. Afterwards cells were incubated as described above, before $1.5 \mathrm{ml}$ of DCmedium replenished with cytokines, was added. Cells were used for further experiments 48 hours after transduction.

Immature and LPS-matured murine BMDCs were seeded at a density of $5 \times 10^{5}$ cells/ well in a 24-well plate in $125 \mu \mathrm{l}$ R10-medium. Virus dilutions at different MOls from 40 to 500 were prepared as described above, using $1 \mathrm{mg} / \mathrm{ml}$ LentiBOOST ${ }^{\circledR}$ and $8 \mu \mathrm{g} / \mathrm{ml}$ Polybrene or buffer only, as described by the manufacturer. After adding $125 \mathrm{\mu l} /$ well virus suspension, BMDCs were incubated as described above, before $1.5 \mathrm{ml}$ of R10-medium was added. Further analyses of transduced BMDCs were performed 48 hours afterwards.

\section{Flow Cytometry.}

Analysis of cells stained with monoclonal antibodies (mAbs; all BioLegend unless specified otherwise) listed below, and 7AAD was performed using a Canto II flow cytometer (BD Bioscience) and FCS Express 5.1 (DeNovo Software). Anti-human mAbs: CD3 (APC, clone UCHT1), CD14 (PE-Cy7, clone 63D3), CD14 (BV510, clone M5E2), CD16 (PE, clone 3G8), CD19 (APC, BD Bioscience, clone HIB19), CD25 (PE-Cy7, clone BC96), CD56 (APC, clone HIB19), CD80 (APC, clone L307.4), CD83 (PE, clone HB15e), CD86 (BV421, clone IT2.2), HLA-DR (APC-Fire, clone L243). Anti-mouse mAbs: CD11c (APC, clone N418), CD25 (APCCy7, clone PC61), CD80 (BV-421, clone 16-10A1), CD83 (PE, clone Michel-19), CD86 (PE-Cy7, clone GL1), IA/I-E (BV510, clone M5/114.15.2), HLA-A0201-PE ELAGIGILTV tetramer (produced in house according to Rodenko et al. $\left.{ }^{41}\right)$.

\section{Cell imaging.}

Cell imaging was performed using the EVOS FL Fluorescence Microscope according to the manufacturer's instructions.

\section{Mixed Lymphocyte Reaction (MLR).}


For MLR assays using human matured DCs, cells were co-cultured at different ratios with $2 \times 10^{6}$ allogeneic human T cells derived from NAF. Co-cultures were incubated in 96-well flat bottom cell culture plates for 72 hours in $200 \mu \mathrm{l}$ of DC medium. For murine mature BMDCs, titrated numbers of cells were cocultured in 96-well flat bottom cell culture plates with $4 \times 10^{5} \mathrm{BALB} / \mathrm{c}$ derived spleen cells for 72 hours in $200 \mu \mathrm{l}$ of R10-medium. As a positive control, human T cells or murine splenic cells only were incubated together with CD3/CD28 Dynabeads in $200 \mu$ of corresponding cell culture medium, whereas NAF only was regarded as negative control. After 3 days, cell-free supernatants of DC-T cell co-cultures were collected and stored at $-20^{\circ} \mathrm{C}$ for further analyses. Cells were pulsed with $3 \mathrm{H}$-thymidine $(1 \mu \mathrm{C} / \mathrm{well}$; PerkinElmer) for 16-20 hours to determine T cell proliferation. Thymidine incorporation was measured using a Wallac 1420 Victor2 Microplate Reader (PerkinElmer).

\section{Cytometric Bead Array.}

Cell culture supernatants were analysed using the LEGENDplex ${ }^{\mathrm{Tw}}$ Human Inflammation Panel 1, or -HU Th Cytokine Panel, or-Mouse Inflammation panel, or -MU Th Cytokine Panel (all BioLegend) according to the manufacturer's instructions.

\section{In vitro RNA transcription and electroporation of human DCs.}

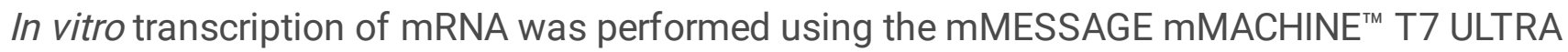

Transcription Kit and purified with an RNeasy Kit, according to the manufacturers' protocols. DCs derived from HLA-A2 positive healthy donors were electroporated with $5 \mu \mathrm{g}$ MelanA mRNA as described before ${ }^{42}$. As a control, mDCs were electroporated without mRNA as described by Gerer et al. ${ }^{42}$.

\section{Priming of CD8 ${ }^{+} \mathrm{T}$ cells.}

For priming of antigen-specific T cells as described in ${ }^{43}$, DCs were transfected as described above with MelanA mRNA, or were peptide pulsed with the MelanA-derived HLA-A2-binding WT peptide EAAGIGILTV. Thawed autologous NAF was co-cultured at a ratio of 1:10 (final concentration $2 \times 10^{5} \mathrm{DCs} / \mathrm{ml}$ and $2 \times 10^{6}$ $\mathrm{NAF} / \mathrm{ml}$ ), in MLPC medium consisting of RPMI 1640, 10\% human serum, $2 \mathrm{mM} \mathrm{L-glutamine,} 20 \mathrm{mg} / \mathrm{L}$ gentamycin, $10 \mathrm{mM}$ HEPES, $1 \mathrm{mM}$ sodium pyruvate and 1\%MEM nonessential amino acids. Fresh MLPC medium was added when necessary. On day 2 and 4, $1000 \mathrm{lU} / \mathrm{ml} \mathrm{IL-2} \mathrm{(Peprotech)} \mathrm{and} 10 \mathrm{ng} / \mathrm{ml} \mathrm{IL-7}$ were supplemented. Cells were harvested after one week of co-culture and stained with an HLA-A0201-PE ELAGIGILTV tetramer, before adding anti-CD3-APC-H7 and anti-CD8-PE-Cy7. Finally, cells were acquired on a FACS Fortessa (BD Bioscience). Resulting data were analyzed using FCS Express 5.1. as follows: Lymphocytes were gated on by forward scatter (FSC) / sideward scatter (SSC), doublets were excluded by 
FSC-area and FSC-height, 7-AAD-positive cells were gated out, $\mathrm{CD} 8^{+}$cells were gated on, and $\mathrm{CD} 3$ vs HLAA tetramer is shown.

\section{Statistical analysis.}

Statistical calculations were performed using GraphPad Prism 8 software.

\section{Ethics statement.}

The generation of human cells from LRSCs of healthy donors was approved by the local ethics committee of the Friedrich-Alexander Universität Erlangen-Nürnberg by the respective positive votes (Re.Nr.: 431_19B and 357_19B). Each participant provided written informed consent. All experiments were carried out in accordance with the WMA Declaration of Helsinki.

The isolation of murine precursor cells to generate BMDC was performed in accordance with the European Communities Council Directive (86/609/EEC; 2010/63/EU) on the Care and Use of Laboratory Animals, and approved by the Government of Lower Franconia - Veterinary Affairs, Consumer Protection, Würzburg, Germany (TS-11/2020). The study was carried out in compliance with the ARRIVE guidelines.

\section{Declarations}

\section{Acknowledgements}

The authors thank the Core Unit 'Cell Sorting and Immunomonitoring' (Kussmaul Campus) for providing access and support for the FACSCantoll-Analyzer and Lena Stich for technical assistance. We also thank Ton Schumacher concerning the production of peptide-HLA-tetramers and for providing the HLAexpression construct. This work was supported by the Deutsche Forschungsgemeinschaft (DFG, German Research Foundation) via the Research Training Group GRK2504, project number 401821119, research project B1, to I.K. Finally, we acknowledge support by the Friedrich-Alexander-Universität ErlangenNürnberg (FAU) within the funding program Open Access Publishing.

\section{Author contributions}

As.S., C.T., J.D., A.S. and I.K. designed the experiments, analysed and interpreted the data and wrote the manuscript. C.T. and S.S. provided the LentiBOOST® substance. As.S., A.D. and T.S. performed experiments and analysed the data. All authors approved the final version of the paper. The present work was performed in fulfilment of the requirements for obtaining the degree Dr. rer. nat. at the FriedrichAlexander-Universität Erlangen-Nürnberg (FAU). 


\section{Additional Information}

CT is co-founder and holds shares of SIRION Biotech GmbH. S.S is employed at SIRION Biotech GmbH. All other authors do not have any potential competing interest.

\section{References}

1. Lee, C. S. et al. Adenovirus-Mediated Gene Delivery: Potential Applications for Gene and Cell-Based Therapies in the New Era of Personalized Medicine. Genes Dis, 4, 43-63 https://doi.org/10.1016/j.gendis.2017.04.001 (2017).

2. Crenshaw, B. J., Jones, L. B., Bell, C. R., Kumar, S. \& Matthews, Q. L. Perspective on Adenoviruses: Epidemiology, Pathogenicity, and Gene Therapy. Biomedicines, 7, https://doi.org/10.3390/biomedicines7030061 (2019).

3. King, R. G. et al. Single-dose intranasal administration of AdCOVID elicits systemic and mucosal immunity against SARS-CoV-2 in mice. bioRxiv, https://doi.org/10.1101/2020.10.10.331348 (2020).

4. Gao, J., Zhang, W. \& Ehrhardt, A. Expanding the Spectrum of Adenoviral Vectors for Cancer Therapy. Cancers (Basel), 12, https://doi.org/10.3390/cancers12051139 (2020).

5. Wei, C. J. et al. Induction of broadly neutralizing H1N1 influenza antibodies by vaccination., 329, 1060-1064 https://doi.org/10.1126/science.1192517 (2010).

6. Alexander, J. et al. Pre-clinical evaluation of a replication-competent recombinant adenovirus serotype 4 vaccine expressing influenza H5 hemagglutinin. PLoS One, 7, e31177 https://doi.org/10.1371/journal.pone.0031177 (2012).

7. Vannucci, L., Lai, M., Chiuppesi, F., Ceccherini-Nelli, L. \& Pistello, M. Viral vectors: a look back and ahead on gene transfer technology. New Microbiol, 36, 1-22 (2013).

8. Alonso-Padilla, J. et al. Development of Novel Adenoviral Vectors to Overcome Challenges Observed With HAdV-5-based Constructs. Mol Ther, 24, 6-16 https://doi.org/10.1038/mt.2015.194 (2016).

9. Reddy, V. S., Natchiar, S. K., Stewart, P. L. \& Nemerow, G. R. Crystal structure of human adenovirus at 3.5 A resolution., 329, 1071-1075 https://doi.org/10.1126/science.1187292 (2010).

10. Liu, H. et al. Atomic structure of human adenovirus by cryo-EM reveals interactions among protein networks., 329, 1038-1043 https://doi.org/10.1126/science.1187433 (2010).

11. Reddy, V. S. \& Nemerow, G. R. Structures and organization of adenovirus cement proteins provide insights into the role of capsid maturation in virus entry and infection. Proc Natl Acad Sci U SA, 111, 11715-11720 https://doi.org/10.1073/pnas.1408462111 (2014).

12. Benevento, M. et al. Adenovirus composition, proteolysis, and disassembly studied by in-depth qualitative and quantitative proteomics. J Biol Chem, 289, 11421-11430 https://doi.org/10.1074/jbc.M113.537498 (2014).

13. Arnberg, N. Adenovirus receptors: implications for targeting of viral vectors. Trends Pharmacol Sci, 33, 442-448 https://doi.org/10.1016/j.tips.2012.04.005 (2012). 
14. Boucher, P., Cui, X. \& Curiel, D. T. Adenoviral vectors for in vivo delivery of CRISPR-Cas gene editors. $J$ Control Release, 327, 788-800 https://doi.org/10.1016/j.jconrel.2020.09.003 (2020).

15. Stein, M. F. et al. Multiple interferon regulatory factor and NF-kappaB sites cooperate in mediating cell-type- and maturation-specific activation of the human CD83 promoter in dendritic cells. Mol. Cell Biol, 33, 1331-1344 doi:MCB.01051-12 [pii];10.1128/MCB.01051-12 [doi] (2013).

16. Tatsis, N. \& Ertl, H. C. Adenoviruses as vaccine vectors. Mol Ther, 10, 616-629 https://doi.org/10.1016/j.ymthe.2004.07.013 (2004).

17. Zhao, J. et al. COVID-19: Coronavirus Vaccine Development Updates. Front Immunol, 11, 602256 https://doi.org/10.3389/fimmu.2020.602256 (2020).

18. Mercier, S. et al. Distinct roles of adenovirus vector-transduced dendritic cells, myoblasts, and endothelial cells in mediating an immune response against a transgene product. $J$ Virol, 76, 28992911 https://doi.org/10.1128/jvi.76.6.2899-2911.2002 (2002).

19. Kaygisiz, K. \& Synatschke, C. V. Materials promoting viral gene delivery. Biomater Sci, 8, 6113-6156 https://doi.org/10.1039/d0bm01367f (2020).

20. Arcasoy, S. M., Latoche, J. D., Gondor, M., Pitt, B. R. \& Pilewski, J. M. Polycations increase the efficiency of adenovirus-mediated gene transfer to epithelial and endothelial cells in vitro. Gene Ther, 4, 32-38 https://doi.org/10.1038/sj.gt.3300349 (1997).

21. Zhao, C. et al. Adenovirus-mediated gene transfer in mesenchymal stem cells can be significantly enhanced by the cationic polymer polybrene. PLoS One, 9, e92908 https://doi.org/10.1371/journal.pone.0092908 (2014).

22. Davis, H. E., Morgan, J. R. \& Yarmush, M. L. Polybrene increases retrovirus gene transfer efficiency by enhancing receptor-independent virus adsorption on target cell membranes. Biophys Chem, 97, 159172 https://doi.org/10.1016/s0301-4622(02)00057-1 (2002).

23. Hofig, I. et al. Poloxamer synperonic F108 improves cellular transduction with lentiviral vectors. $J$ Gene Med, 14, 549-560 https://doi.org/10.1002/jgm.2653 (2012).

24. Simon, B. et al. Enhancing lentiviral transduction to generate melanoma-specific human T cells for cancer immunotherapy. J Immunol Methods, 472, 55-64 https://doi.org/10.1016/j.jim.2019.06.015 (2019).

25. Becker, M., Cotena, A., Gordon, S. \& Platt, N. Expression of the class A macrophage scavenger receptor on specific subpopulations of murine dendritic cells limits their endotoxin response. Eur $J$ Immunol, 36, 950-960 https://doi.org/10.1002/eji.200535660 (2006).

26. Ulyanova, T. et al. VCAM-1 expression in adult hematopoietic and nonhematopoietic cells is controlled by tissue-inductive signals and reflects their developmental origin., 106, 86-94 https://doi.org/10.1182/blood-2004-09-3417 (2005).

27. Ansari, A. M. et al. Cellular GFP Toxicity and Immunogenicity: Potential Confounders in in Vivo Cell Tracking Experiments. Stem Cell Rev Rep, 12, 553-559 https://doi.org/10.1007/s12015-016-9670-8 (2016). 
28. Skelton, D., Satake, N. \& Kohn, D. B. The enhanced green fluorescent protein (eGFP) is minimally immunogenic in C57BL/6 mice. Gene Ther, 8, 1813-1814 https://doi.org/10.1038/sj.gt.3301586 (2001).

29. Gambotto, A. et al. Immunogenicity of enhanced green fluorescent protein (EGFP) in BALB/c mice: identification of an H2-Kd-restricted CTL epitope. Gene Ther, 7, 2036-2040 https://doi.org/10.1038/sj.gt.3301335 (2000).

30. Stripecke, R. et al. Immune response to green fluorescent protein: implications for gene therapy. Gene Ther, 6, 1305-1312 https://doi.org/10.1038/sj.gt.3300951 (1999).

31. Re, F., Srinivasan, R., Igarashi, T., Marincola, F. \& Childs, R. Green fluorescent protein expression in dendritic cells enhances their immunogenicity and elicits specific cytotoxic T-cell responses in humans. Exp Hematol, 32, 210-217 https://doi.org/10.1016/j.exphem.2003.10.014 (2004).

32. Mangeot, P. E. et al. High levels of transduction of human dendritic cells with optimized SIV vectors. Mol Ther, 5, 283-290 https://doi.org/10.1006/mthe.2002.0541 (2002).

33. Rouas, R. et al. Lentiviral-mediated gene delivery in human monocyte-derived dendritic cells: optimized design and procedures for highly efficient transduction compatible with clinical constraints. Cancer Gene Ther, 9, 715-724 https://doi.org/10.1038/sj.cgt.7700500 (2002).

34. Huang, S., Endo, R. I. \& Nemerow, G. R. Upregulation of integrins alpha v beta 3 and alpha $v$ beta 5 on human monocytes and T lymphocytes facilitates adenovirus-mediated gene delivery. J Virol, 69, 2257-2263 https://doi.org/10.1128/JVI.69.4.2257-2263.1995 (1995).

35. Schneider, S. D., Rusconi, S., Seger, R. A. \& Hossle, J. P. Adenovirus-mediated gene transfer into monocyte-derived macrophages of patients with X-linked chronic granulomatous disease: ex vivo correction of deficient respiratory burst. Gene Ther, 4, 524-532 https://doi.org/10.1038/sj.gt.3300432 (1997).

36. Burke, B., Sumner, S., Maitland, N. \& Lewis, C. E. Macrophages in gene therapy: cellular delivery vehicles and in vivo targets. $J$ Leukoc Biol, 72, 417-428 (2002).

37. Lutz, M. B. et al. An advanced culture method for generating large quantities of highly pure dendritic cells from mouse bone marrow. J. Immunol. Methods, 223, 77-92 doi:S0022-1759(98)00204-X [pii] (1999).

38. Zal, T., Volkmann, A. \& Stockinger, B. Mechanisms of tolerance induction in major histocompatibility complex class II-restricted T cells specific for a blood-borne self-antigen. J. Exp. Med, 180, 20892099 (1994).

39. Pfeiffer, I. A. et al. Leukoreduction system chambers are an efficient, valid, and economic source of functional monocyte-derived dendritic cells and lymphocytes. Immunobiology 218, 1392-1401, doi:S0171-2985(13)00140-X [pii];10.1016/j.imbio. 2013.07.005 [doi] (2013).

40. He, T. C. Adenoviral vectors. Curr. Protoc. Hum. Genet Chap. 12, Unit, doi:10.1002/0471142905.hg1204s40 [doi] (2004).

41. Rodenko, B. et al. Generation of peptide-MHC class I complexes through UV-mediated ligand exchange. Nat Protoc, 1, 1120-1132 https://doi.org/10.1038/nprot.2006.121 (2006). 
42. Gerer, K. F., Hoyer, S., Dorrie, J. \& Schaft, N. Electroporation of mRNA as Universal Technology Platform to Transfect a Variety of Primary Cells with Antigens and Functional Proteins. Methods Mol Biol, 1499, 165-178 https://doi.org/10.1007/978-1-4939-6481-9_10 (2017).

43. Bosch, N. C. et al. NF-kappaB activation triggers NK-cell stimulation by monocyte-derived dendritic cells. Ther Adv Med Oncol, 11, 1758835919891622 https://doi.org/10.1177/1758835919891622 (2019).

\section{Figures}

a

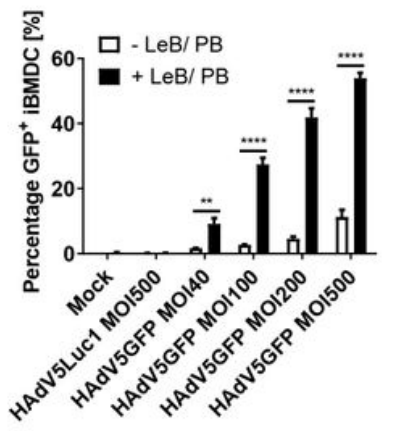

C

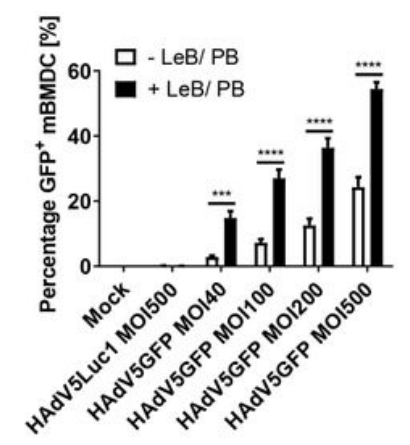

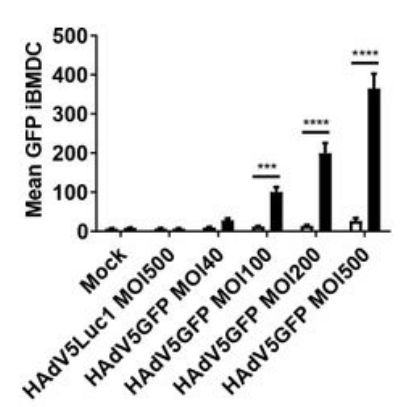

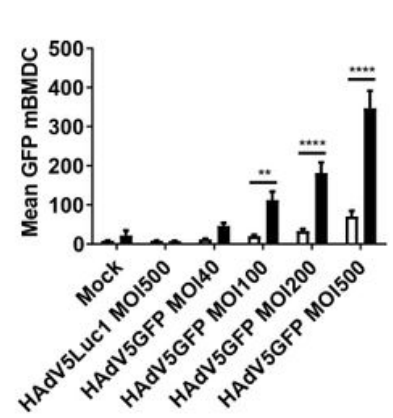

b ¿

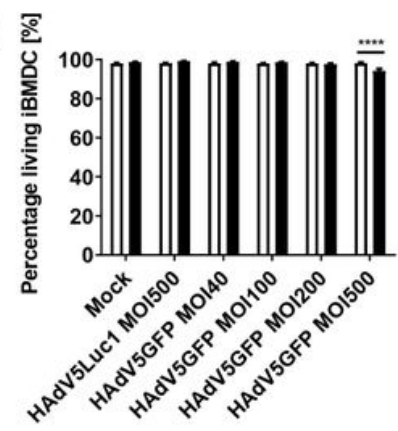

d $\stackrel{8}{\stackrel{8}{8}}$

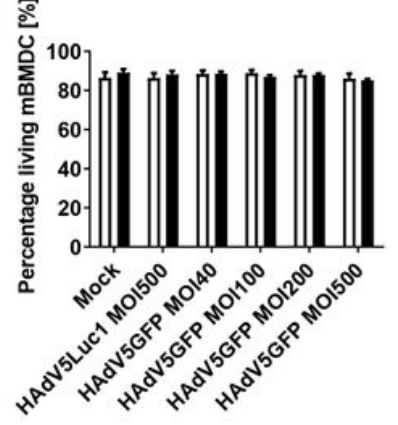

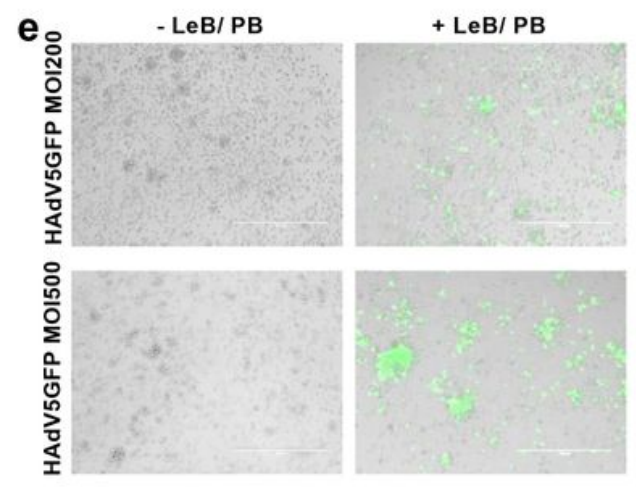

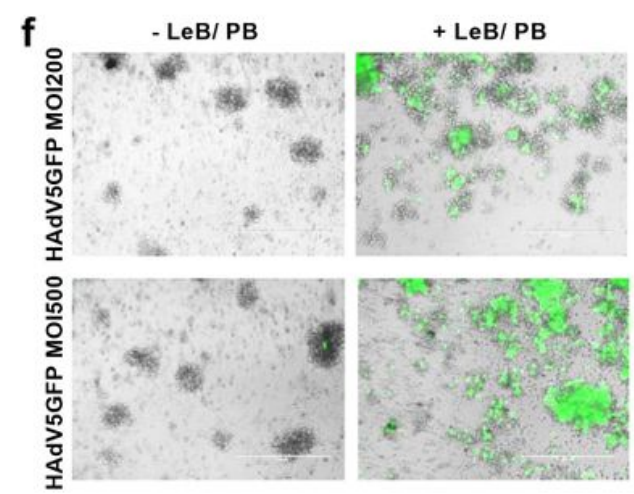

Figure 1

\section{Figure 1}

LentiBOOST ${ }^{8} /$ Polybrene highly increase transduction efficacy of murine BMDCs. Immature (i) and LPSmatured (m)BMDCs were transduced with HAdV5GFP at different MOls in combination with LentiBOOST ${ }^{\circledR} /$ Polybrene (+LeB/ PB) or with water (control), for 48 hours. As a control, cells were mocktransduced ("Mock") or transduced using HAdV5Luc1. (a, c) Flow cytometric analyses of iBMDCs (a) and 
mBMDCs. (c) GFP expression presented as percentage and mean fluorescence of GFP+ BMDCs. (b, d) Flow cytometric analyses of iBMDCs (b) and mBMDCs (d) regarding 7AAD- living cells. (a - d) Data are mean \pm SEM of four independent experiments with cells derived from different mice. Two-way ANOVA and Sidak correction were performed. $* * \mathrm{P}<0.01$, $* \star * \mathrm{P}<0.001$, $* \star \star * \mathrm{P}<0.0001$, bars without annotation are not significant $(P>0.05)$ in comparison to the respective condition water control $(e, f)$ Fluorescence imaging of HAdV5GFP-transduced iBMDCs (e) and mBMDCs (f) 48 hours after transduction. One representative experiment out of four is shown.

a
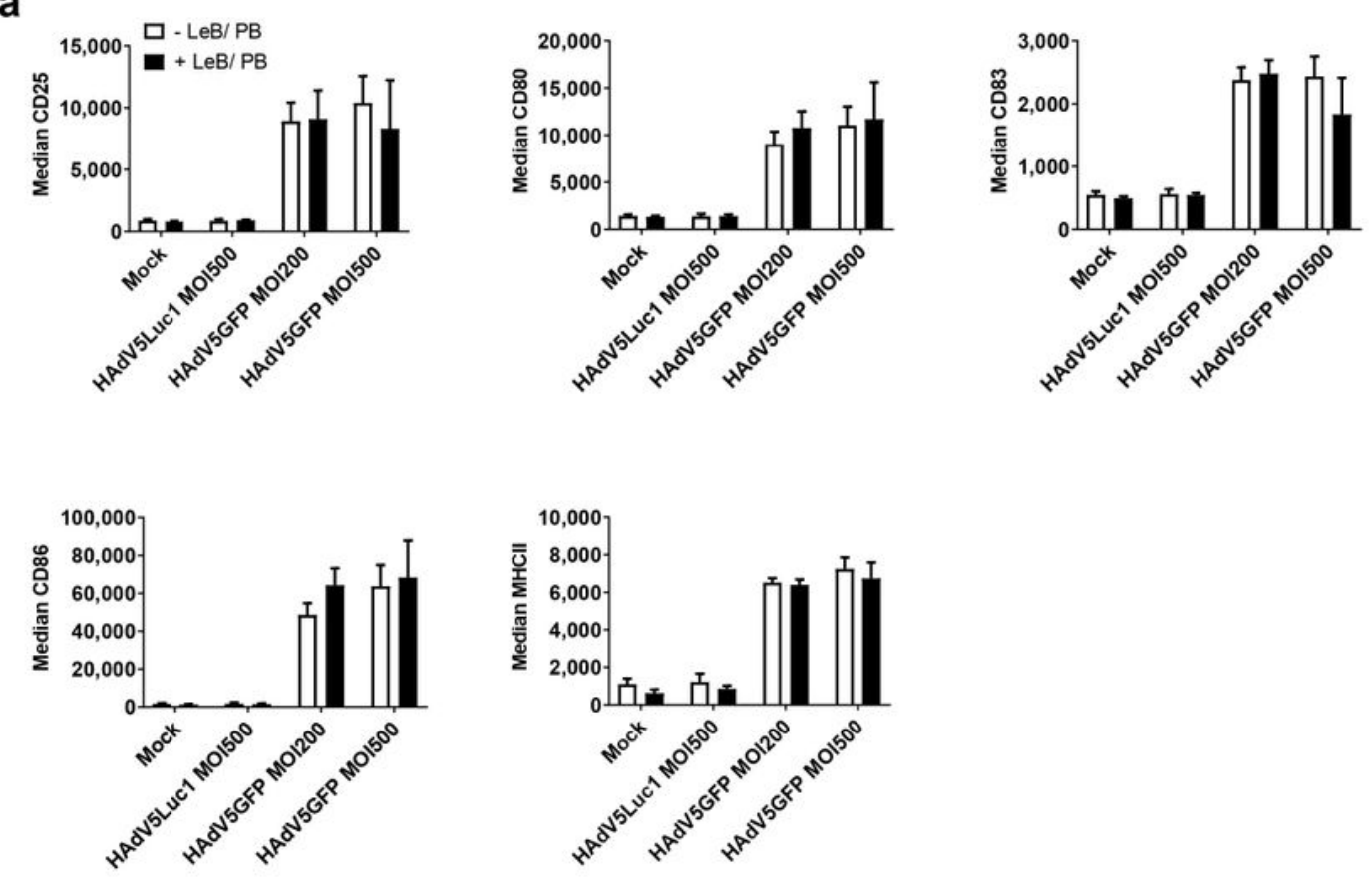

b
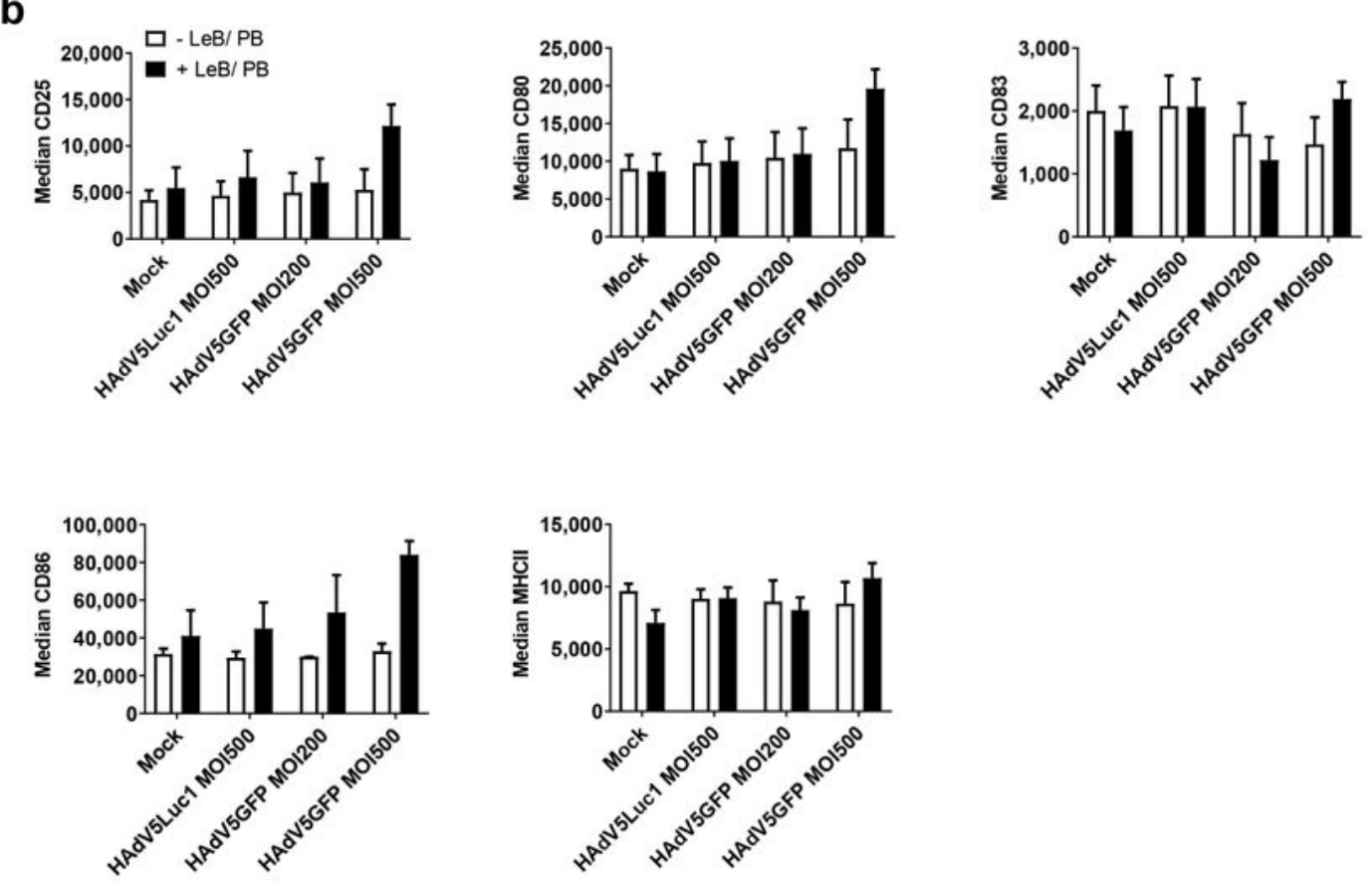

Figure 2 
Figure 2

LentiBOOST ${ }^{\circledR} /$ Polybrene does not impair the maturation status of BMDCs. $(a, b)$ Immature and LPSmatured BMDCs were transduced with HAdV5Luc1 at a MOI of 500, or with HAdV5GFP at a MOI of 200 and 500 in combination with LentiBOOST $B /$ Polybrene (+LeB/ PB) or a water control, for 48 hours. As a negative control, cells were mock transduced ("Mock"). Flow cytometric analyses regarding CD25-, CD80-, CD86- and MHC-II expression, of iBMDCs (a) and mBMDCs (b), presented as median fluorescent intensity of positive cells. Results are shown as mean \pm SEM of three independent experiments with cells derived from different mice. Two-way ANOVA and Sidak correction were performed. Bars without annotation are not significant $(P>0.05)$ in comparison to the respective condition without $(-)$ LeB and PB.

a
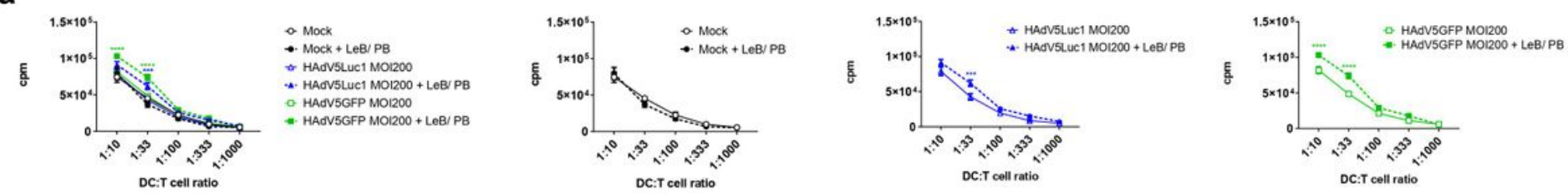

b
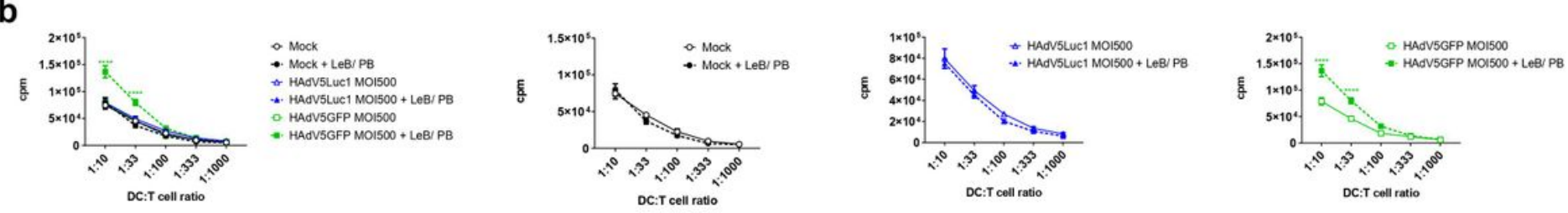

C
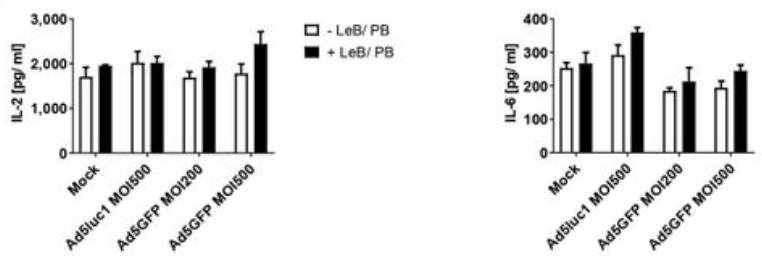

d
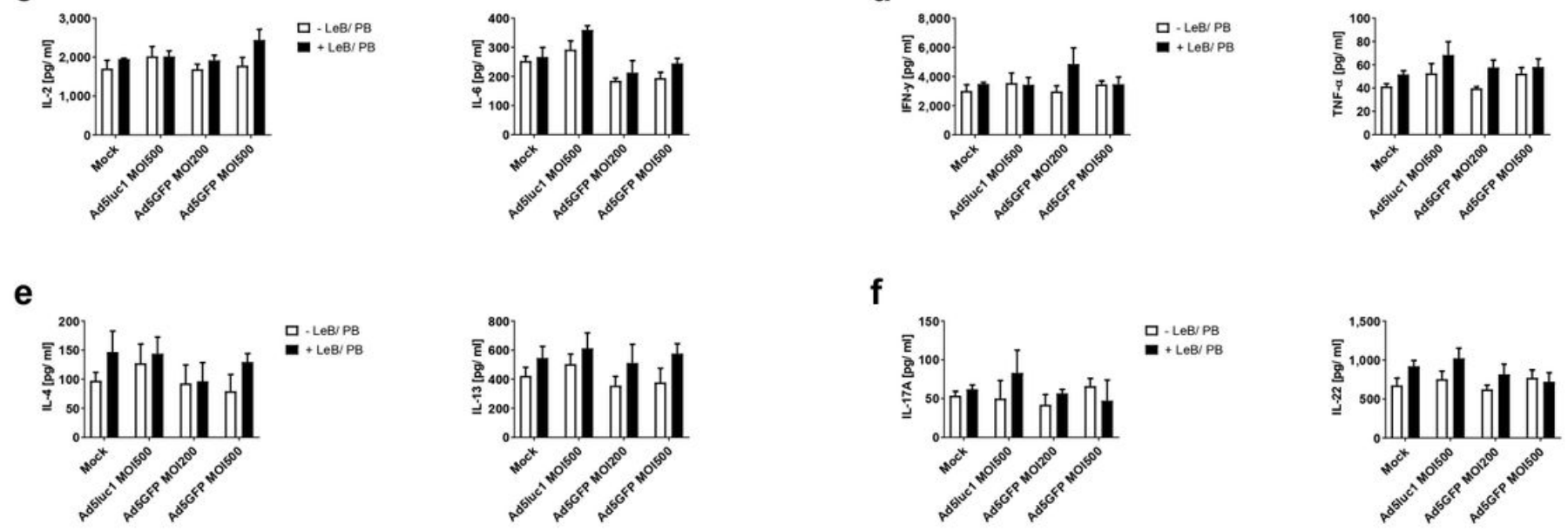

Figure 3

\section{Figure 3}

HAdV5 transduced mBMDCs in combination with LentiBOOST®/ Polybrene retain their function. LPSmatured BMDCs were transduced with HAdV5Luc1 or HAdV5GFP at an MOI of 200 or 500 in combination with LentiBOOST ${ }^{\circledR} /$ Polybrene (+LeB/ PB) or water (-LeB/ PB). Fourty-eight hours afterwards, cells were 
assessed using an allogeneic mixed lymphocyte reaction assay. $(a, b)$ Allogeneic mixed lymphocyte reactions (MLRs) of mBMDCs transduced at MOI200 (a) or MOI500 (b). Cells were co-cultured with BALB/c derived spleen cells at the indicated ratios for $72 \mathrm{~h}$, before pulsing with $1 \mu \mathrm{C} /$ well [H3]-thymidine. Thymidine uptake was assessed using a 1450 MicroBeta counter. (c - $f$ ) Cytometric bead array (CBA) of MLR-derived cell culture supernatants. Cell-free supernatants of the DC-T cell co-cultures described in ( $a$, b), were collected at day 3 before pulsing with thymidine and stored at $-20^{\circ} \mathrm{C}$. Content of cytokines was determined by cytometric bead array. $(a-f)$ Data are mean \pm SEM of three independent experiments with cells derived from different mice. Two-way ANOVA and Sidak correction were performed. ${ }^{\star} \star * \quad P<0.001$, $\star \star \star \star ~ P<0.0001$, bars without annotation are not significant $(P>0.05)$ in comparison to the respective condition - LeB/ PB.
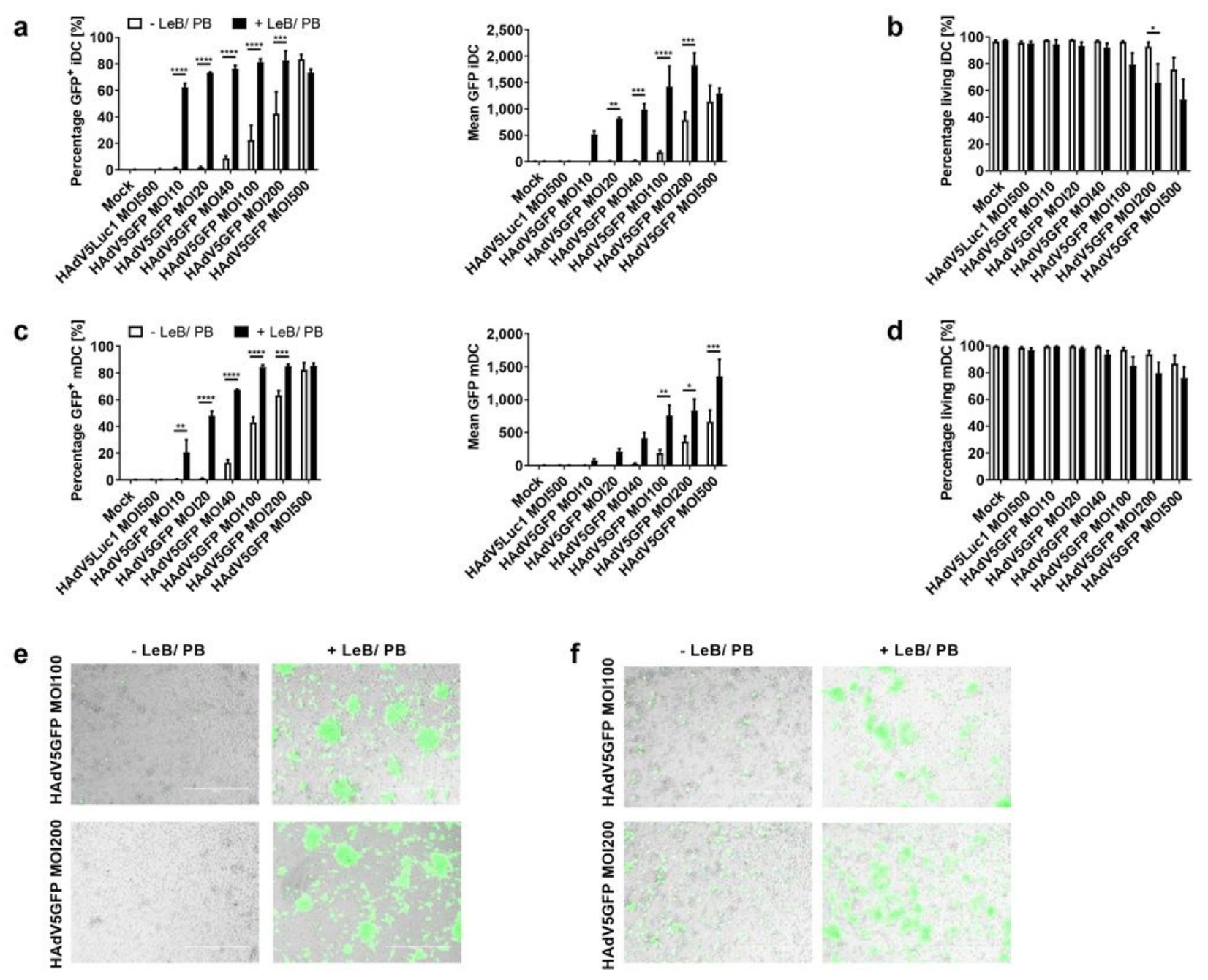

Figure 4

\section{Figure 4}

LentiBOOST $\circledast /$ Polybrene enables high transduction rates of immature and mature human DCs with HAdV5. Human monocyte-derived immature (iDC) and mature (m)DCs were transduced with HAdV5Luc1 or HAdV5GFP at indicated MOls, ranging from MOI 10 to 50 in combination with LentiBOOST ${ }^{\circledR}$ / Polybrene (+LeB/ PB) or water only (-LeB/ PB). As a negative control, cells were not transduced with adenovirus ("Mock"). Forty-eight hours afterwards, cells were analysed for expression of GFP and 
survival. (a - d) Flow cytometric analyses of iDCs $(a, b)$ and mDCs $(c, d)$ regarding their GFP expression (a, c) depicted as percentage positive cells and median fluorescent intensity, as well as on 7AAD- living cells (b, d). Data are mean \pm SEM of three independent experiments with cells derived from different healthy

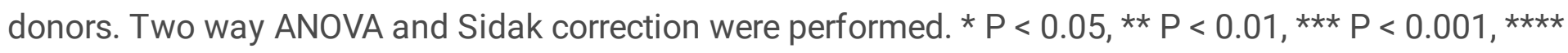
$P<0.0001$, bars without annotation are not significant $(P>0.05)$ in comparison to the respective condition - LeB/ PB. (e, f) Fluorescence imaging of iDCs (e) and mDCs (f) two days after transduction with HAdV5GFP. One representative experiment out of three is shown.

a
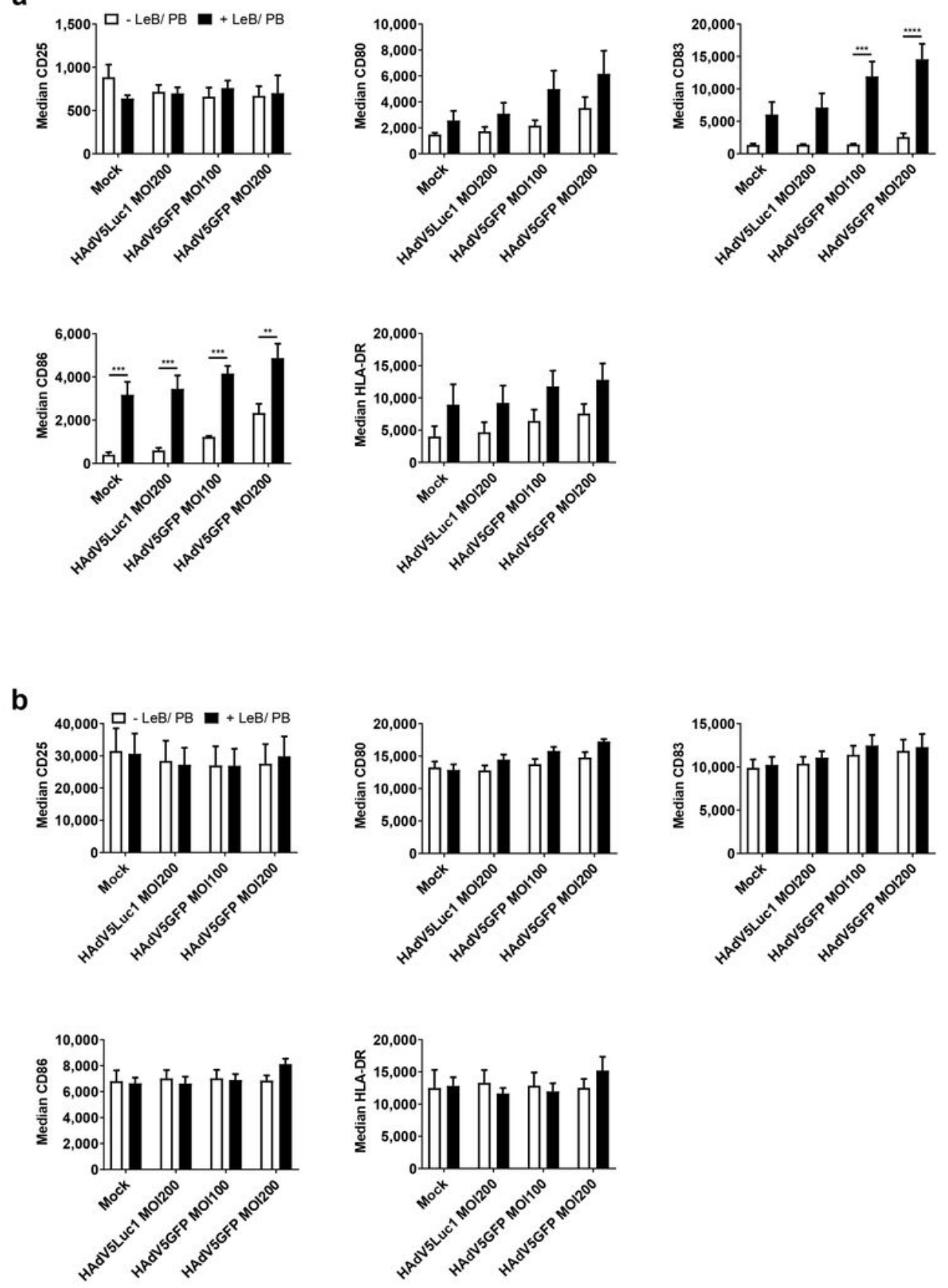

Figure 5 


\section{Figure 5}

Induction of maturation in immature DCs following adenoviral transduction in the presence of LentiBOOST $\AA /$ Polybrene. $(a, b)$ Human monocyte-derived iDCs and mDCs were transduced with HAdV5Luc1 at a MOI of 200, or with HAdV5GFP at a MOI of 100 and 200 in combination with LentiBOOST ${ }^{\circledR} /$ Polybrene (+LeB/ PB) or water (-LeB/ PB), for 48 hours. Afterwards, median fluorescent intensity of CD25, CD80, CD83, CD86 and HLA-DR was determined by flow cytometry for iDCs (a) and $\mathrm{mDC}(\mathrm{b})$. Data are mean \pm SEM of four independent experiments with cells derived from different healthy

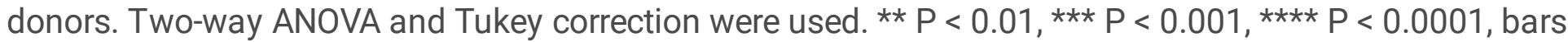
without annotation are not significant $(P>0.05)$ in comparison to the respective condition $-\mathrm{LeB} / \mathrm{PB}$.

a
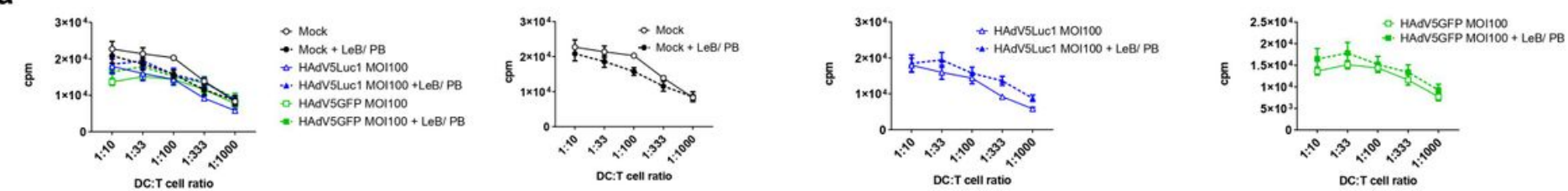

b
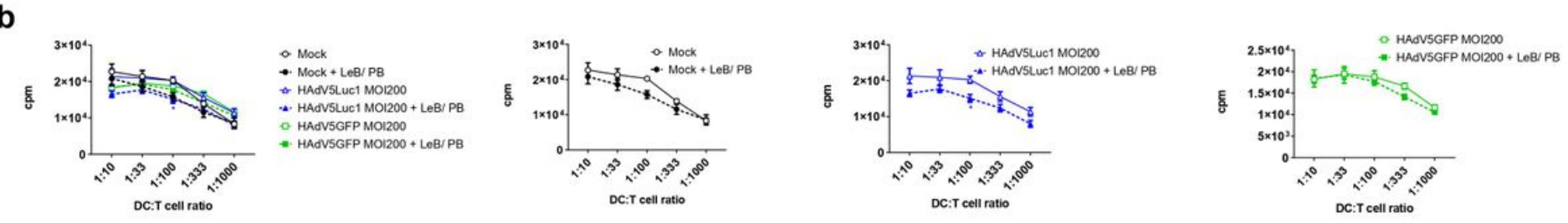
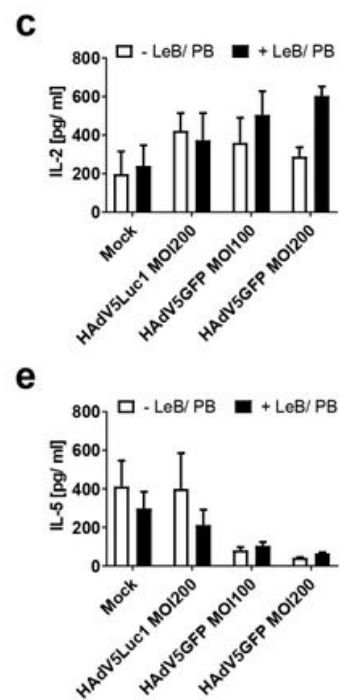
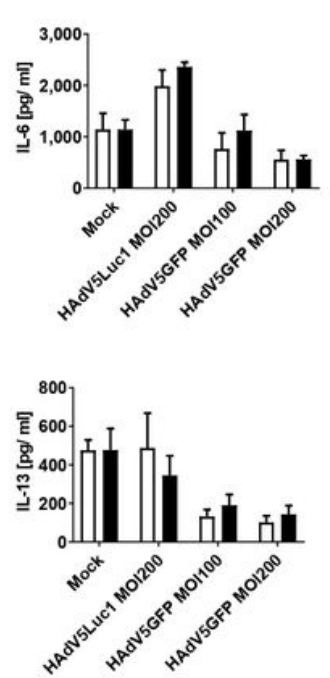

d

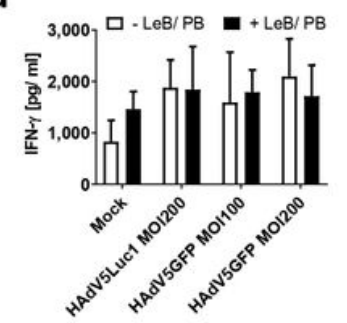

f

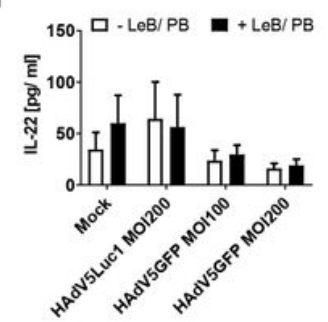

Figure 6

\section{Figure 6}

Enhanced adenoviral transduction efficacy using LentiBOOST®/ Polybrene retains the functionality of human mDCs. Mature dendritic cells were transduced with HAdV5Luc1 or HAdV5GFP at a MOI of 100 and 200 in combination with LentiBOOST $\AA /$ Polybrene (+LeB/ PB) or water only (-LeB/ PB), for 48 hours. $(\mathrm{a}, \mathrm{b})$ Allogeneic MLR assays with mature DCs transduced with HAdV5Luc1/ HAdV5GFP at a MOI of 100 (a) and a MOI of 200 (b). Mature DCs and T cells, derived from the non-adherent fraction (NAF) of 
peripheral blood mononuclear cells (PBMCs), were co-cultivated at the indicated ratios for $72 \mathrm{~h}$ and subsequently pulsed with $1 \mu \mathrm{C} /$ well [H3]-thymidine for $16 \mathrm{~h}$. Thymidine uptake was assessed using a 1450 MicroBeta counter. ( $c$ - $f$ ) Cell-free supernatants derived from MLR assays described in $(a, b)$ were analysed by CBA for their content of cytokines, 72 hours after co-culture. $(a-f)$ Data are mean \pm SEM of three independent experiments with cells derived from different healthy donors. Two-way ANOVA and Sidak correction were performed. ${ }^{*} P<0.05$, bars without annotation are not significant $(P>0.05)$ in comparison to the respective condition $-\mathrm{LeB} / \mathrm{PB}$.
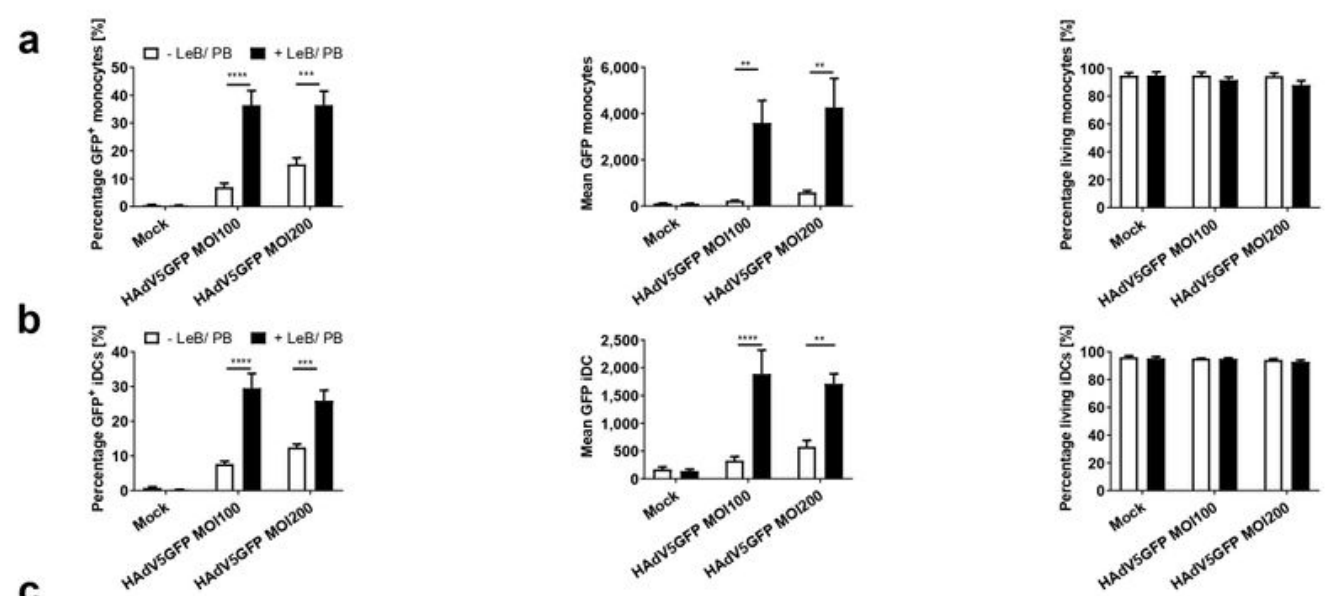
C
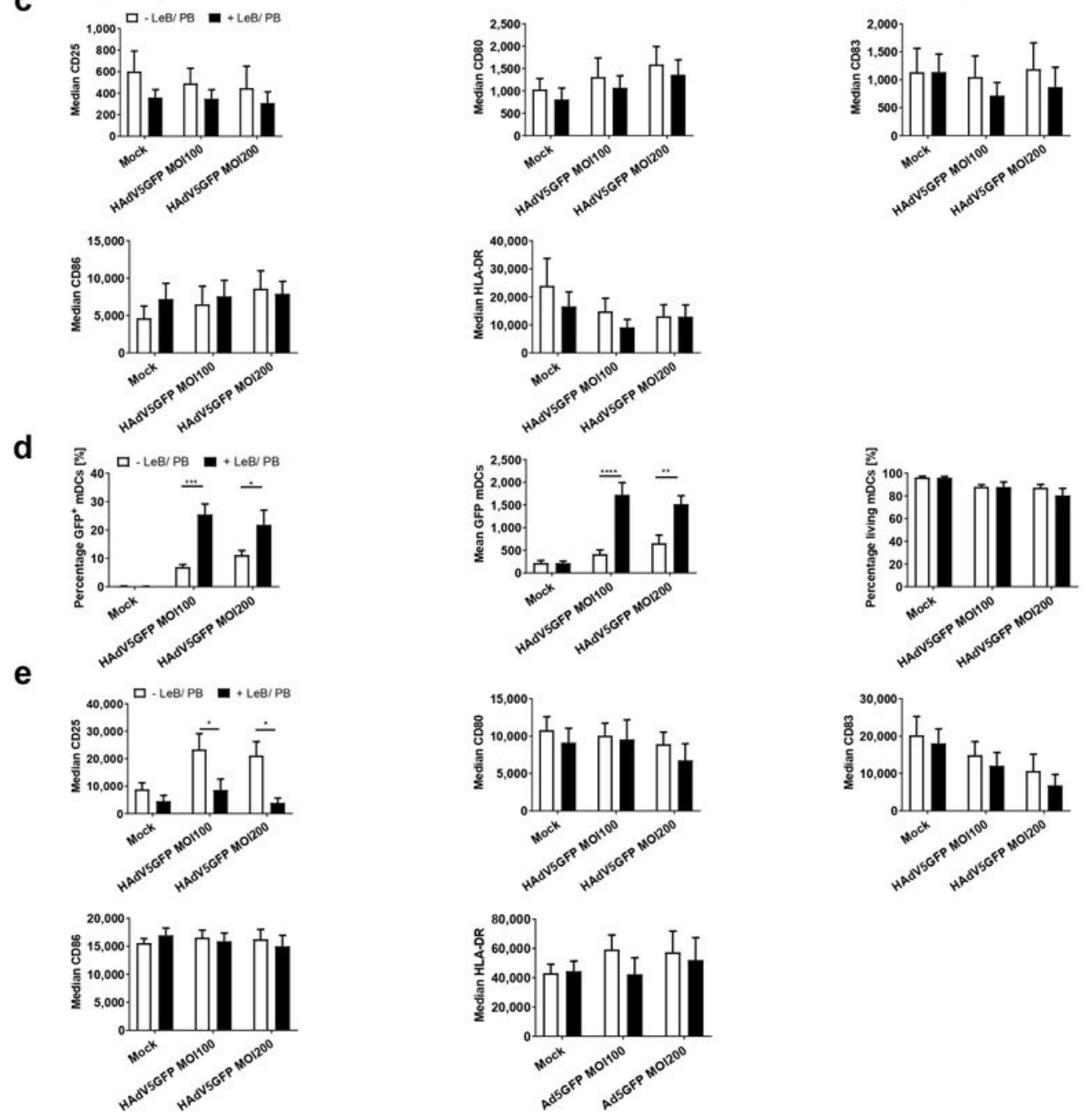

Figure 7 


\section{Figure 7}

Human monocytes efficiently transduced in the presence of LentiBOOST $\AA /$ Polybrene can be differentiated into iDCs and mDCs. Human monocytes were transduced with HAdV5GFP at a MOI of 100 and 200 in combination with LentiBOOST $® /$ Polybrene (+LeB/ PB) or water only (-LeB/ PB). Non-infected cells ("Mock") served as a negative control. Monocytes were either immediately analysed by flow cytometry or differentiated into iDCs for 4 days using GM-CSF and IL-4, or into day $5 \mathrm{mDCs}$, additionally adding IL-1 $\beta$, IL-6, PGE2 and TNF- $a$ for 24 hours. $(a, b, d)$ Flow cytometric analyses on the percentage and median fluorescent intensity of GFP positive as well as on 7-AAD negative living CD14+ HLA-DR+ monocytes (a), CD11c+ HLA-DR+ iDCs (b), or CD11c+ HLA-DR+ mDCs (c). (c, d) Flow cytometric analyses on cell surface markers CD25, CD80, CD83, CD86 and HLA-DR on iDCs (c) and mDCs (e). (a - e) Data are mean \pm SEM of five independent experiments with cells derived from different healthy donors. Two-way

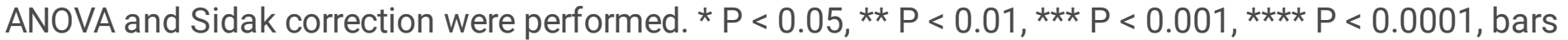
without annotation are not significant $(P>0.05)$ in comparison to the respective condition - LeB/ $P B$.

a
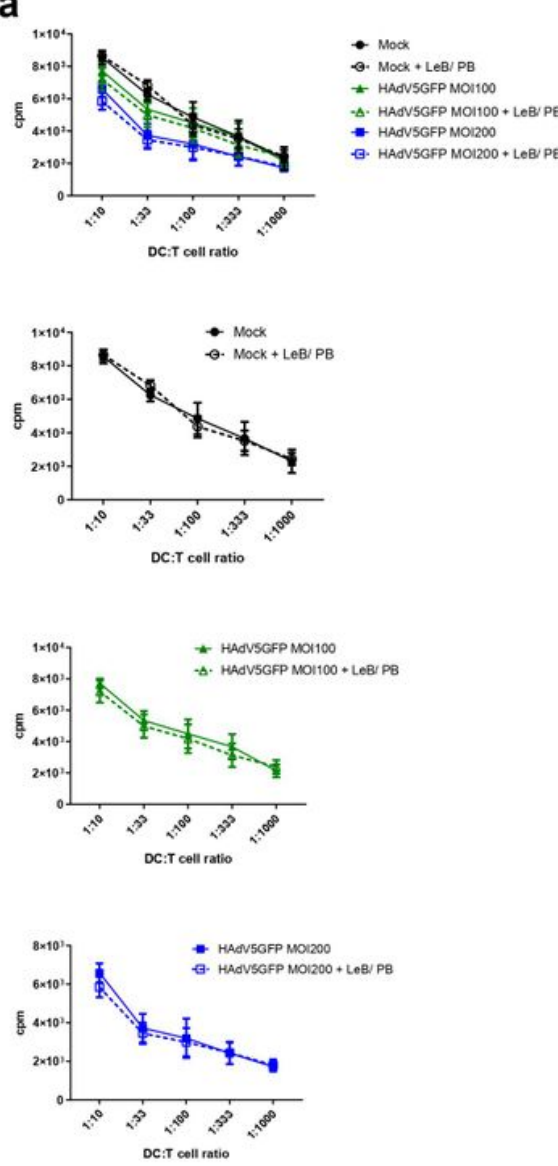

b

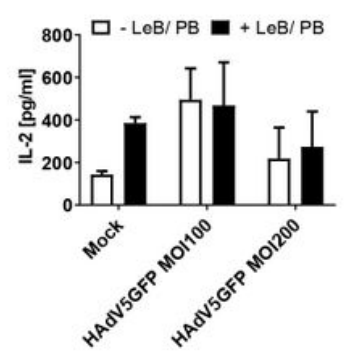

d
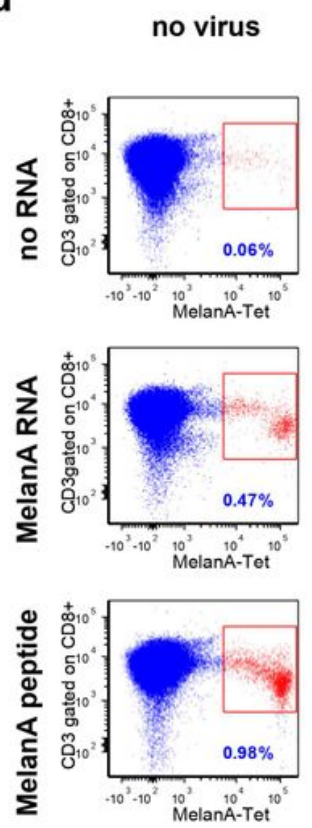

C

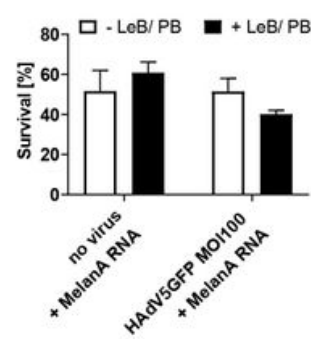

no virus+LeB/PB
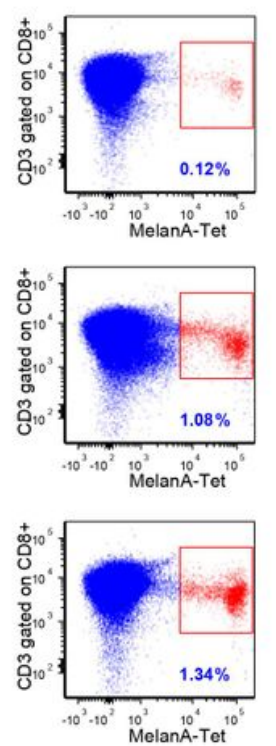

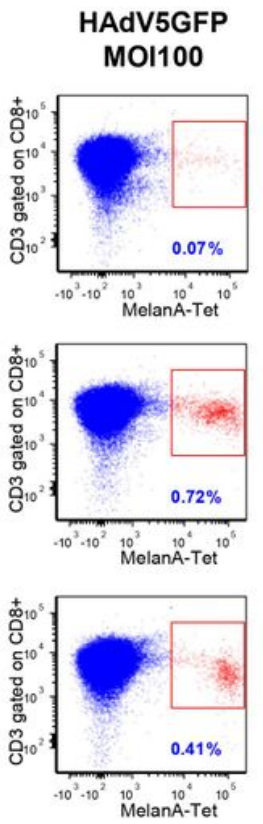

HAdV5GFP MOI100+LeB/ PB
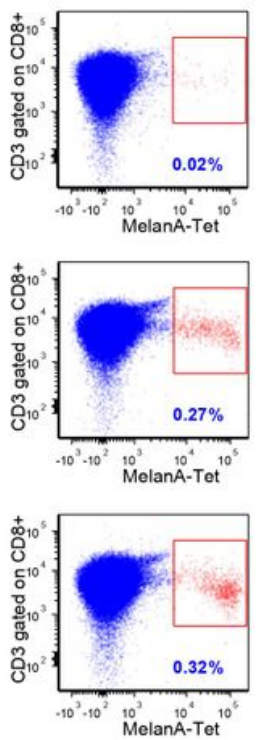

Figure 8 
Mature DCs differentiated from transduced monocytes in the presence of LentiBOOST®/ Polybrene are fully functional. Human monocytes were mock-treated ("Mock" or "non virus") or transduced with HAdV5GFP (MOI100 or MOI200) either in the presence of buffer only (-LeB/ PB) or LentiBOOST®/ Polybrene (+LeB/ PB) as described before. Afterwards, cells were differentiated into mDCs. (a) Mixed lymphocyte reaction (MLR) assays. Mature DCs and allogeneic T cells were co-cultivated at the indicated ratios for $72 \mathrm{~h}$ and subsequently pulsed with $1 \mu \mathrm{C} /$ well [H3]-thymidine for $16 \mathrm{~h}$. Thymidine uptake was assessed using a 1450 MicroBeta counter. (b) Cell-free supernatants derived from MLR assays described in (a), were analysed by CBA for their content of IL-2. (c, d) Priming of autologous T cells in a tumourantigen-specific manner. Mature DCs derived from transduced monocytes were either electroporated with RNA coding for MelanA, or loaded with a MelanA-derived peptide (EAAGIGILTV), for $1 \mathrm{~h}$. Dendritic cells electroporated without RNA ("no RNA") served as a control. (c) Survival of non-transduced-, or HAdV5GFP transduced mDCs either in the presence or absence of LentiBOOST $\AA /$ Polybrene was assessed before and 4 hours after electroporation with MelanA RNA by trypan blue staining. Percentage of living cells after electroporation compared to living cells before electroporation is shown. Data are mean \pm SEM of four $(a, b)$ or three (c) independent experiments with cells derived from different healthy donors. Two-way ANOVA and Sidak correction were performed. Bars without annotation are not significant $(P>0.05)$ in comparison to the respective condition -LeB/ PB. (d) Four hours after electroporation, these DCs were used to stimulate autologous NAF cells at a 1:10 ratio. One week after stimulation, the percentage of MelanA-specific CD3+ CD8+ T cells (depicted in red) was analysed by tetramer-staining and flowcytometry. Indicated percentages refer to the total number of CD8+ T cells. Shown here is one representative experiment out of three.

\section{Supplementary Files}

This is a list of supplementary files associated with this preprint. Click to download.

- Stracketal.SupplementaryInformationAmendments2082021.pdf 